\title{
Inclusive chemical characterization of tourmaline: Mössbauer study of Fe valence and site occupancy
}

\author{
M. Darby Dyar, ${ }^{1, *}$ Marjorie E. Taylor, ${ }^{1}$ Timothy M. Lutz, ${ }^{1}{ }^{\text {Carl A. Francis, }}{ }^{2}$ \\ Charles V. Guidotti, ${ }^{3}$ and Michael Wise ${ }^{4}$ \\ ${ }^{1}$ Department of Geology and Astronomy, West Chester University, West Chester, Pennsylvania 19383, U.S.A. \\ ${ }^{2}$ Harvard Mineralogical Museum, 24 Oxford St., Cambridge, Maine 02138, U.S.A. \\ ${ }^{3}$ Department of Geological Sciences, University of Maine, Orono, Maine 04469, U.S.A. \\ ${ }^{4}$ Department of Mineral Sciences NHB 119, National Museum of Natural History, Washington, D.C. 20560, U.S.A.
}

\begin{abstract}
We report here the results of a series of inclusive chemical characterizations, including all elements except oxygen, for a suite of 54 tourmaline samples. A combination of analytical techniques was used to analyze for major and light elements (electron microprobe), $\mathrm{Fe}^{3+}$ and $\mathrm{Fe}^{2+}$ (Mössbauer spectroscopy), $\mathrm{H}$ (U extraction), and $\mathrm{B}, \mathrm{Li}$, and $\mathrm{F}$ (ion microprobe, or SIMS). The B content of the tourmalines studied ranges from 2.86 to $3.26 \mathrm{~B}$ per formula unit (pfu) with 31 anions; excess boron is believed to reside in the $\mathrm{Si}$ site. Li ranges from 0.0 to 1.44 $\mathrm{Li} \mathrm{pfu}$ and $\mathrm{F}$ contents are $0.0-0.91 \mathrm{pfu}$. $\mathrm{H}$ contents range from nearly anhydrous up to 3.76 $\mathrm{H}$ pfu and do not correlate simply with $\mathrm{Fe}^{3+}$ content.

Mössbauer results show that tourmaline exhibits the entire range of $\mathrm{Fe}^{3+} / \Sigma \mathrm{Fe}$ from 0.0-1.0. $\mathrm{Fe}^{2+}$ is represented in the spectra by three doublets, with occupancy in at least three distinct types of Y sites (with different types of nearest and next nearest neighbors). $\mathrm{Fe}^{3+}$ was found in 26 of the 54 samples studied. Although Mössbauer data do not allow the distinction between ${ }^{[1} \mathrm{Fe}^{3+}$ and ${ }^{[\mathrm{Z}} \mathrm{Fe}^{3+}$ site occupancies to be made, XRD data on these samples suggest that the majority of $\mathrm{Fe}^{3+}$ is also in Y. Of the samples studied, ${ }^{[4]} \mathrm{Fe}^{3+}$ occurs in nine; five of those were either olenite or uvite with extensive Na substitution. A mixed valence doublet corresponding to delocalized electrons shared between adjacent octahedra was observed in 14 of the samples studied. Projection pursuit regression analysis shows that distribution of $\mathrm{Fe}$ among doublets is a function (albeit a complex one) of bulk composition of the tourmaline and supports the interpretation of doublets representing different populations of neighbors. Variations in $\mathrm{Fe}^{3+}$ / $\mathrm{Fe}^{2+}$ ratio cannot be directly related to variations in charge in any single site of the structure. $\mathrm{Fe}^{3+} / \mathrm{Fe}^{2+}$ ratio is probably controlled by the prevailing oxidation state in the bulk rock assemblage, rather than by any particular crystal chemical substitution.
\end{abstract}

\section{INTRODUCTION}

In the years before the introduction of instrumental methods for analysis of minerals, it was common practice to analyze minerals by wet chemistry, evaluating all constituents that might be present. However, the ease of access and simplicity of analyses made possible by the electron microprobe, coupled with the demise of most wet chemical facilities, has made contemporary mineralogy increasingly dependent on an analytical tool that generates only partial chemical compositions for minerals. Although advances in the detection of light elements through use of synthetic crystals have made electron microprobes capable of estimating their abundances (McGee and Anovitz 1996), the analysis of elements lighter than fluorine remains problematic, even for the ion microprobe (Hervig 1996). For minerals in which light elements are significant chemical components, it is difficult to accurately and comprehensively evaluate their crystal chemistry.

For no mineral is this task more problematic than for tourmaline. Previous wet chemical analyses of tourmaline published in, for example, Deer et al. (1986) show con-

*E-mail: mddyar@amherst.edu

0003-004X/98/0708-0848\$05.00

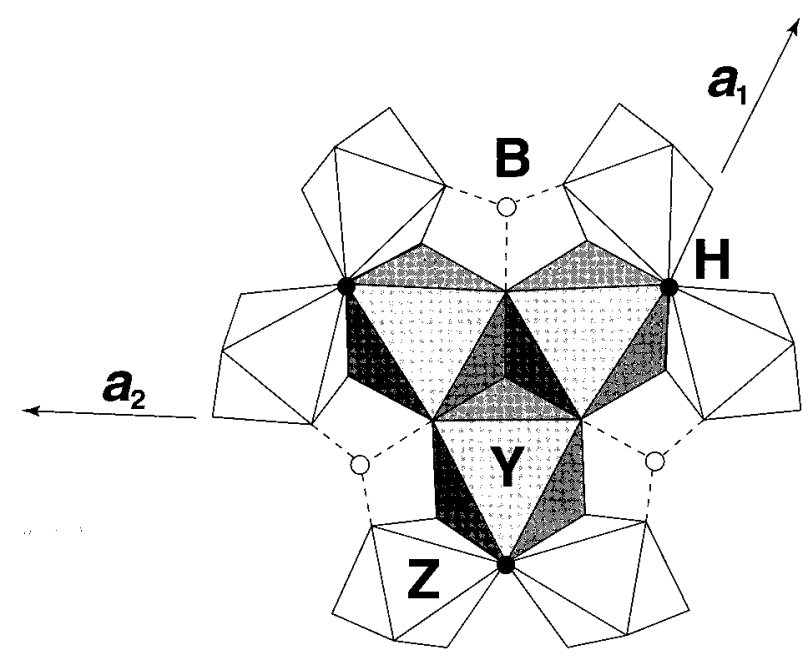

Figure 1. View down the $c$ axis of tourmaline, showing the juxtaposition of $\mathrm{Y}$ (shaded) and $\mathrm{Z}$ (unshaded) octahedra to the $\mathrm{B}$ sites. Corners of all octahedra are $\mathrm{O}$ atoms (shared); $\mathrm{OH}^{-}$positions are indicated by small, filled circles at the appropriate locations. B sites are shown as open circles, with dashed lines connecting B atoms to the corners of adjacent octahedra. The $\mathrm{Z}$ sites link to form three-membered rings (not shown). 

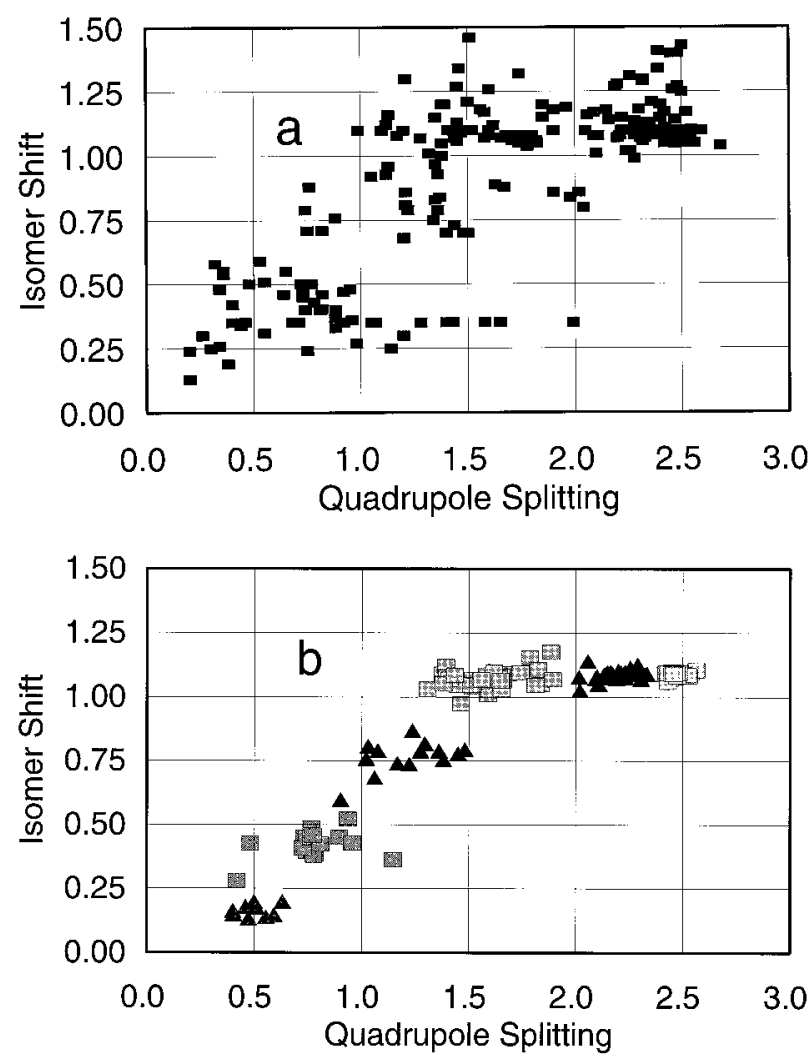

Figure 2. Comparison of literature values of the hyperfine parameters I.S. (isomer shift) and Q.S. (quadrupole splitting) for Mössbauer spectra of tourmaline (a) and data from the current study (b), all expressed in $\mathrm{mm} / \mathrm{s}$. Symbols in (b) represent ${ }^{[4}$ or 6$] \mathrm{Fe}^{3+}$, ${ }^{[6]} \mathrm{Fe}^{3+}, \mathrm{ED},{ }^{\left[{ }^{3} 3\right.} \mathrm{Fe}^{2+},{ }^{\left[{ }^{2}\right]} \mathrm{Fe}^{2+}$, and ${ }^{\left[{ }^{1} 1\right]} \mathrm{Fe}^{2+}$ from left to right. Literature data come from Burns (1972), Belov et al. (1972, 1973), Hermon et al. (1973), Pollak and Bruyneel (1974), Alverez et al. (1975), Dambly et al. (1976), Gorelikova et al. (1978), Scorzelli et al. (1976), Korovushkin et al. (1979), Saegusa et al. (1979), Mattson and Rossman (1984), Kraczka et al. (1986), Ferrow et al. (1988), Ferrow (1993, 1994), Fuchs et al. (1995), Linares et al. (1996), Pieczka and Kraczka (1997) and Pieczka et al. (1997). All data represent fits to room temperature spectra, cited with respect to the midpoint of $\mathrm{Fe}$ metal. It is apparent from these results that our data, which were collected on the same type of apparatus and, perhaps more importantly, fit with the same model, show less scatter than the collected data from the literature.

siderable variation of all chemical components in its structure. Despite the existence of these data, recent workers have consistently reported (or assumed for convenience) that $\mathrm{B}$, in particular, is stoichiometric in tourmaline. The present study was initiated, in part, to test the validity of this assumption, and more generally, to evaluate the full extent of variation in tourmaline chemistry using the best possible analytical techniques for each element in its composition. Note that we have not analyzed these samples for $\mathrm{O}$ although the technology for doing so is certainly available using fast neutron activation analysis (e.g., Volborth and Banata 1963; McKlveen
TABLE 1. Previous site assignments

\begin{tabular}{|c|c|c|c|c|c|}
\hline $\begin{array}{c}\text { Site } \\
\text { and } \\
\text { valence }\end{array}$ & $\begin{array}{c}\text { Nearest } \\
\text { neighbor }\end{array}$ & $\begin{array}{c}\text { Next nearest } \\
\text { neighbor }\end{array}$ & $\begin{array}{l}\text { Isomer } \\
\text { shift } \\
(\delta)\end{array}$ & $\begin{array}{l}\text { Quadru- } \\
\text { pole } \\
\text { split- } \\
\text { ting } \\
(\Delta)\end{array}$ & Ref \\
\hline${ }^{[} \mathrm{MFe}^{2+}$ & & & 1.08 & 2.52 & $a$ \\
\hline [2] $\mathrm{Fe}^{2+}$ & & & 1.09 & 1.56 & a \\
\hline [2] $\mathrm{Fe}^{3+}$ & & & 0.39 & 0.95 & a \\
\hline${ }^{[3]} \mathrm{Fe}^{3+}$ & & & 0.37 & 0.64 & $\mathrm{a}$ \\
\hline $\mathrm{Fe}^{2 \cdot n+}$ & & & 0.72 & 1.35 & \\
\hline$\left[{ }^{[}\right] \mathrm{Fe}^{2+}$ & $\mathrm{O}_{4}(\mathrm{OH})_{2}$ & $\mathrm{R}^{2+} \mathrm{R}^{2+}$ & 1.08 & 2.54 & b \\
\hline [V] $\mathrm{Fe}^{2+}$ & $\mathrm{O}_{4}(\mathrm{OH})_{2}$ & $\mathrm{R}^{2+} \mathrm{R}^{3+}$ & 1.08 & 2.37 & b \\
\hline [1] $\mathrm{Fe}^{2+}$ & $\mathrm{O}_{4}(\mathrm{OH}) \mathrm{F}$ & $\mathrm{R}^{2+}\left(\mathrm{R}^{2+}, \mathrm{Fe}^{3+}\right)$ & 1.08 & 2.11 & b \\
\hline$\left.{ }^{[}\right] \mathrm{Fe}^{2+}$ & $\mathrm{O}_{4}(\mathrm{OH})_{2}$ & $\mathrm{R}^{2+} \mathrm{Ti}^{\mathrm{j}^{+}}$ & 1.08 & 2.11 & b \\
\hline$\left[{ }^{[}\right] \mathrm{Fe}^{2+}$ & $\mathrm{O}_{4}(\mathrm{OH}) \mathrm{F}$ & $\mathrm{R}^{2+}\left(\mathrm{Al}, \mathrm{Ti}^{4+}\right)$ & 1.08 & 1.68 & b \\
\hline$\left[{ }^{2}\right] \mathrm{Fe}^{2+}$ & $\mathrm{O}_{5}(\mathrm{OH})$ & $\mathrm{R}^{2+}\left(\mathrm{R}^{2+}, \mathrm{R}^{3+}, \mathrm{Ti}^{4+}\right)$ & 1.08 & 1.68 & b \\
\hline$\left[\mathrm{M}^{2} \mathrm{Fe}^{3+}\right.$ & $(\mathrm{OH})_{4}(\mathrm{OH}, \mathrm{F}, \mathrm{O})_{2}$ & ??? & 0.35 & 0.46 & $\mathrm{~b}$ \\
\hline
\end{tabular}

Note: $\mathrm{a}=$ Burns (1972) and Saegusa (1979). b = Pieczka et al. (1997). Parameters are in millimeters per second.

1981). However, the interpretation of weight percent $O$ data is problematic because it depends on assumptions of constant cation site occupancy in order for results to be normalized. For further discussion of this problem, see Dyar et al. (1991).

A second motivation for this study is the recent recognition of the ubiquitous occurrence of B-bearing minerals in a wide variety of igneous, metamorphic, and sedimentary parageneses (London et al. 1996; Grew 1996; Henry and Dutrow 1996). Development of accurate thermodynamic models to express phase relations in B-bearing systems requires very detailed understanding of the chemical relationships in the constituent minerals (Anovitz and Hemingway 1996). This project seeks to evaluate the predominant controlling substitutions and potential thermodynamic end-members with which the tourmaline structure might be modeled. Again, such a task requires detailed chemical characterization of every element present in the structure at abundances greater than trace amounts.

Finally, we have undertaken this study to evaluate the effectiveness and appropriateness of various technologies other than the electron microprobe for mineral analysis. Accordingly, we utilized the electron microprobe for major element analysis; the ion microprobe (secondary ionization mass spectrometer, or SIMS) for $\mathrm{B}, \mathrm{Li}$, and $\mathrm{F}$; Mössbauer spectroscopy for $\mathrm{Fe}^{3+} / \Sigma \mathrm{Fe}$; and uranium extraction for $\mathrm{H}_{2} \mathrm{O}$. Although the latter two methods were employed on bulk samples (powders) for the present project, microscale methods for those analytical problems are currently under development or are already in use. [For example, microscale $\mathrm{Fe}^{3+} / \mathrm{Fe}^{2+}$ determinations on hornblende (Delaney et al. 1996), clinopyroxene, and other silicate minerals (Dyar et al. 1996; Delaney et al. 1998) are made using synchrotron micro-XANES. Microscopic measurements of $\mathrm{H}$ are routinely done by PIGE analysis at the State University of New York accelerator lab in Albany (Lanford 1992).] In this study, we hope to demonstrate the viability of the inclusive approach, and show 
TABLE 2. Sample descriptions

\begin{tabular}{|c|c|c|c|c|}
\hline $\begin{array}{l}\text { Sample } \\
\text { number }\end{array}$ & Source & $\begin{array}{l}\text { Museum } \\
\text { number }\end{array}$ & Type & Description and references \\
\hline 1. & HS & 108796 & Dravite & $\begin{array}{l}8 \times 6 \times 5 \mathrm{~cm} \text { abraded black crystal from Madagascar (Frondel et al. } \\
1966 ; \text { Hermon et al. 1973). }\end{array}$ \\
\hline 2. & HS & 112566 & Schorl & $\begin{array}{l}\text { black nodule from the Alto Lighona pegmatite field, Zambezia, Mozam- } \\
\text { bique. }\end{array}$ \\
\hline 3. & HS & 98144 & Elbaite & black striated "pocket" crystal from a pegmatite, Minas Gerais, Brazil. \\
\hline 4. & HS & 108491 & Dravite & $\begin{array}{l}\text { black radiating crystals forming a sphere with secondary } \mathrm{Cu} \text { minerals } \\
\text { from the Lucero mine, Chile. }\end{array}$ \\
\hline 5. & HS & 86321 & Uvite & $\begin{array}{l}\text { colorless crystals in coarse marble, DeKalb, St. Lawrence Co., New } \\
\text { York. }\end{array}$ \\
\hline 6. & $\mathrm{HS}$ & 44061 & Schorl & $\begin{array}{l}\text { "aphrizite" lustrous, black euhedra to } 5 \mathrm{~mm} \text { in granite from the Harz } \\
\text { Mtns., Germany. }\end{array}$ \\
\hline 7. & HS & 98695 & Schorl & Aucohuma, Sorata, Bolivia. \\
\hline 8. & $\mathrm{HS}$ & 112547 & Foitite & $\begin{array}{l}2 \mathrm{~cm} \text { brown cross fiber vein in quartz from a wolframite deposit at Cop- } \\
\text { per Mountain, Picuris district, Taos Co., New Mexico. }\end{array}$ \\
\hline 9. & WSU & Silver Knob & Dravite & $\begin{array}{l}\text { Vanadian, from quartz-graphite schist roof pendant at Silver Knob, Mar- } \\
\text { iposa Co., California (Snetsinger 1966; Foit and Rosenberg 1979). }\end{array}$ \\
\hline 10. & WSU & Jack Creek & Schorl & $\begin{array}{l}\text { zoned crystals with dumortierite in metasomatized quartz latite, Jack } \\
\text { Creek, Jefferson Co., Montana (Foit et al. 1989; Foit 1989). }\end{array}$ \\
\hline 11. & CMN & 49356 & Dravite & York River (Grice and Ercit 1993). \\
\hline 12. & CMN & 43293 & Buergerite & $\begin{array}{l}\text { from rhyolite at Mexquitic, San Luis Potosi, Mexico (Mason et al. 1964; } \\
\text { Donnay et al. 1966; Barton 1969; Tippe and Hamilton 1971; Hermon } \\
\text { et al. 1973; Grice and Ercit 1993). }\end{array}$ \\
\hline 13. & CMN & 32008 & Dravite & Tait Farm (Grice and Ercit 1993). \\
\hline 14. & CMN & 43873 & Dravite & Pierrepont (Grice and Ercit 1993). \\
\hline 15. & CMN & 43230 & Dravite & Yinnietharra, Australia (Grice and Ercit 1993). \\
\hline 16. & CMN & 52210 & Uvite & Hall Farm (Grice and Ercit 1993). \\
\hline 17. & RK & PresFracFill & Schorl & Preston Fracture Fill, western Canada (King 1990). \\
\hline 18. & RK & McKen Isle & Schorl & McKenzie Island, western Canada (King 1990). \\
\hline 19. & RK & Highway 527 & Schorl & Highway 527, western Canada (King 1990). \\
\hline 20. & RK & Sigma-3 & Schorl & Sigma-3, western Canada (King 1990). \\
\hline 21. & RK & Bevcon no. 1 & Schorl & Bevcon \#1, western Canada (King 1990). \\
\hline 22. & RK & Ghost Lake & Schorl & Ghost Lake, western Canada (King 1990). \\
\hline 23. & this study & O-T16-92 & Dravite & $\begin{array}{l}\text { from metapelite at lower sillimanite grade, Dead River Fm., west slope } \\
\text { of Bald Mt., Rangeley, Oxford Co., Maine. }\end{array}$ \\
\hline 24. & this study & Ru-T17-92 & Dravite & $\begin{array}{l}\text { from melanosome bands in the migmatitic Noisy Brook gneiss, Roxbu- } \\
\text { ry, Oxford Co., Maine. }\end{array}$ \\
\hline 25. & this study & Ru-T18-92 & Dravite & $\begin{array}{l}\text { in metapelite adjacent to contact with pegmatite at sillimanite grade in } \\
\text { Small Falls Fm., Rumford, Oxford Co., Maine. }\end{array}$ \\
\hline 26. & this study & SMFALLS & Dravite & Small Falls pegmatite, Rumford, Oxford Co., Maine. \\
\hline 27. & this study & DLUX1 & Schorl & $\begin{array}{l}\text { from a granitic pegmatite associated with the Sebago Granite North } \\
\text { Windham, Oxford Co., Maine. }\end{array}$ \\
\hline 28. & this study & RT12 & Schorl & $\begin{array}{l}\text { from fine to medium grained Sebago Granite, North Windham, Oxford } \\
\text { Co., Maine. }\end{array}$ \\
\hline 29. & HS & 131955 & Schorl & black porphyroblasts in white schist, Newry, Oxford Co., Maine. \\
\hline 30. & HS & LCW2356 & Draviate & $\begin{array}{l}\text { porphyroblasts in amphibolite contact metamorphosed by granitic peg- } \\
\text { matite, Dunton Mine, Newry, Oxford Co., Maine (Henry and Dutrow } \\
\text { 1990). }\end{array}$ \\
\hline 31. & HS & LCW1067 & Schorl & in cleavelandite, Dunton Mine, Newry, Oxford Co., Maine. \\
\hline 32. & HS & 126022 & Schorl & coexisting with spodumene, Nevel Quarry, Newry, Oxford Co., Maine. \\
\hline 33. & this study & Whitecap1 & Schorl & Whitecap Mt., western Maine. \\
\hline 34. & this study & STRGR-2 & Schorl & Streaked Mountain, STRGR-2, western Maine. \\
\hline 35. & this study & SEBGR-2 & Schorl & Sebago Granite SEBGR-2, western Maine. \\
\hline 36. & USNM & BMT-47 & Schorl & $\begin{array}{l}\text { black, exocontact, Black Mt. Pegmatite, Rumford, Oxford Co., Maine } \\
\text { (Brown and Wise 1991). }\end{array}$ \\
\hline 37. & USNM & BMT-47 & Elbaite & $\begin{array}{l}\text { green, wall rock, Black Mt. Pegmatite, Rumford, Oxford Co., Maine } \\
\text { (Brown and Wise 1991). }\end{array}$ \\
\hline 38. & USNM & BMT-3 & Schorl & $\begin{array}{l}\text { wall rock, Black Mt. Pegmatite, Rumford, Oxford Co., Maine (Brown } \\
\text { and Wise 1991). }\end{array}$ \\
\hline 39. & USNM & BMT-75 & Schorl & $\begin{array}{l}\text { wall rock, Black Mt. Pegmatite, Rumford, Oxford Co., Maine (Brown } \\
\text { and Wise 1991). }\end{array}$ \\
\hline 40. & USNM & BMT-B & Elbaite & $\begin{array}{l}\text { green, 1st intermediate zone, Black Mt. Pegmatite, Rumford, Oxford } \\
\text { Co., Maine (Brown and Wise 1991). }\end{array}$ \\
\hline 41. & USNM & BMT-33 & Dravite & $\begin{array}{l}\text { black, 1st intermediate zone, Black Mt. Pegmatite, Rumford, Oxford } \\
\text { Co., Maine (Brown and Wise 1991). }\end{array}$ \\
\hline 42. & USNM & BMT-11 & Elbaite & $\begin{array}{l}\text { dark blue, clevelandite unit, 1st intermediate zone, Black Mt. Pegmatite, } \\
\text { Rumford, Oxford Co., Maine (Brown and Wise 1991). }\end{array}$ \\
\hline 43. & USNM & BMT-20 & Elbaite & $\begin{array}{l}\text { dark blue, clevelandite unit, 1st intermediate zone, Black Mt. Pegmatite, } \\
\text { Rumford, Oxford Co., Maine (Brown and Wise 1991). }\end{array}$ \\
\hline 44. & USNM & BMT-65 & Schorl & $\begin{array}{l}\text { blue-green, 2nd intermediate zone, Black Mt. Pegmatite, Rumford, Ox- } \\
\text { ford Co., Maine (Brown and Wise 1991). }\end{array}$ \\
\hline 45. & USNM & BMT-17 & Olenite & $\begin{array}{l}\text { pink, lepidolite pod in 2nd intermediate zone, Black Mt. Pegmatite, } \\
\text { Rumford, Oxford Co., Maine (Brown and Wise 1991). }\end{array}$ \\
\hline
\end{tabular}


TABLE 2-Continued

\begin{tabular}{|c|c|c|c|c|}
\hline $\begin{array}{l}\text { Sample } \\
\text { number }\end{array}$ & Source & $\begin{array}{l}\text { Museum } \\
\text { number }\end{array}$ & Type & Description and references \\
\hline 46. & USNM & BMT-19 & Olenite & $\begin{array}{l}\text { pink, lepidolite pod in 2nd intermediate zone, Black Mt. Pegmatite, Rum- } \\
\text { ford, Oxford Co., Maine (Brown and Wise 1991). }\end{array}$ \\
\hline 47. & USNM & BMT-A & Olenite & $\begin{array}{l}\text { pink, core, Black Mt. Pegmatite, Rumford, Oxford Co., Maine (Brown and } \\
\text { Wise 1991). }\end{array}$ \\
\hline 48. & USNM & BMT-49 & Elbaite & $\begin{array}{l}\text { green, 2nd intermediate zone, Black Mt. Pegmatite, Rumford, Oxford Co., } \\
\text { Maine (Brown and Wise 1991). }\end{array}$ \\
\hline 49. & PN & HP 2-1 & Schorl & Harney Peak Granite, Custer Co., South Dakota (Rockhold et al. 1987). \\
\hline 50. & PN & HP 5-1 & Schorl & Harney Peak Granite, Custer Co., South Dakota (Rockhold et al. 1987). \\
\hline 51. & PN & HP 6-1 & Schorl & Harney Peak Granite, Custer Co., South Dakota (Rockhold et al. 1987). \\
\hline 52. & HS & 108749 & Schorl & Raglan Twp., Renfrew Co., Ontario, Canada. \\
\hline 53. & HS & 18796 & Dravite & associated with corundum from Macon Co., North Carolina. \\
\hline 54. & HS & 12146 & Dravite & $\begin{array}{l}\text { in contact marble with grossular, spinel, xanthophyllite, Davis Gulch, near } \\
\text { Helena. Montana. }\end{array}$ \\
\hline
\end{tabular}

Note: Samples loaned from: HS = Harvard Mineralogical Museum; WSU = Washington State University; CMN = Canadian Museum of Nature; RK $=$ Robert Kerrich; USNM = U.S. National Museum, Smithsonian; PN = Peter Nabelek.

that application of these techniques (even in bulk samples) merits further work toward their development into more widely available tools with microanalytical capabilities for mineralogical studies.

Various aspects of this work are discussed in several papers. Here, we present information on all the analytical methods used, and tabulate the complete cation compositions of all 54 tourmaline samples in the suite. The discussion focusses on the results of the Mössbauer spectroscopy study of these samples, to examine the site occupancy and valence state of $\mathrm{Fe}$ in tourmaline. The effects of composition and crystal chemistry on the valence state and site partitioning of $\mathrm{Fe}$ in each tourmaline are studied. A second contribution (Lutz et al., in preparation) will use statistical analysis of the data to extract and examine the prevalent substitutions represented in the entire data set. A third paper (Dyar et al., in preparation) will focus on the analytical difficulties encountered in the light element analysis of the samples presented here, discussing the advantages and disadvantages of the analytical methods used for light elements. Results of an interlaboratory comparison of tourmaline analyses by different methods are presented. It should also be noted that single-crystal X-ray diffraction studies of a subset of the minerals presented here are underway (John Hughes, personal communication).

\section{BACKGROUND}

Extensive variation in composition and cell dimensions occurs in tourmaline group minerals due to the substitution of cations of varying sizes and electrical charges into three crystallographic positions. The simplified chemical formula found in Deer et al. (1986), $\mathrm{XY}_{3} \mathrm{Z}_{6} \mathrm{~B}_{3} \mathrm{Si}_{6} \mathrm{O}_{27}$ $(\mathrm{OH})_{3}(\mathrm{OH}, \mathrm{F})$, where $\mathrm{X}=\mathrm{Na}, \mathrm{Ca}, \mathrm{K}$, and $\square, \mathrm{Y}=\mathrm{Mg}$, $\mathrm{Fe}, \mathrm{Mn}, \mathrm{Li}, \mathrm{Cr}^{3+}, \mathrm{V}^{3+}, \mathrm{Ti}$, and $\mathrm{Al}$ and $\mathrm{Z}=\mathrm{Al}, \mathrm{Mg}, \mathrm{Fe}$, $\mathrm{V}^{3+}$ and $\mathrm{Cr}^{3+}$ will be used here. Clearly, the mineral tourmaline presents a challenge to chemical analysis due to the diversity and multiplicity of its elements.

Tourmaline accommodates $\mathrm{Fe}^{2+}$ and $\mathrm{Fe}^{3+}$ in both the $\mathrm{Y}$ and $\mathrm{Z}$ octahedral sites (Fig. 1). These two sites differ from each other in size and symmetry. The Y octahedron shares edges with two $\mathrm{Y}$ and two $\mathrm{Z}$ octahedra and is relatively larger (polyhedral volume is $10.715 \AA^{3}$; Smyth and Bish 1988) than the smaller $Z$ octahedron (polyhedral volume is $1.929 \AA^{3}$; Smyth and Bish 1988), which shares three edges with two $\mathrm{Z}$ and one $\mathrm{Y}$ octahedra.

$\mathrm{Fe}$ in each valence and site experiences a distinct electromagnetic distortion that is discernible through Mössbauer spectroscopy. Thus, the Mössbauer spectrum of a $\mathrm{Fe}^{2+}$ - and $\mathrm{Fe}^{3+}$-bearing tourmaline might be expected to have a total of four possible doublets corresponding to ${ }^{[Y]} \mathrm{Fe}^{2+},{ }^{[\mathrm{Z}]} \mathrm{Fe}^{2+},{ }^{\left[{ }^{\gamma}\right]} \mathrm{Fe}^{3+}$, and ${ }^{[\mathrm{Z}]} \mathrm{Fe}^{3+}$, as identified by Burns (1972) and Simon (1973) in the first Mössbauer studies of tourmaline. Another possibility is ${ }^{[4]} \mathrm{Fe}^{3+}$, presumably in low $\mathrm{Si}$ and $\mathrm{Al}$ samples. In addition, some tourmaline spectra are even more complicated because the edge sharing of the octahedral sites promotes electron sharing. If the total Fe content of the tourmaline is high enough or ordered appropriately, $\mathrm{Fe}$ atoms in adjacent sites can share electrons, making possible the presence of electron charge delocalization doublets (ED) in the Mössbauer spectra. These represent the averaged valence states of the Fe atoms that are sharing electrons, a number somewhere between $2+$ and $3+$. ED doublets were first reported in tourmaline spectra by Saegusa et al. (1979), and Ferrow et al. (1988) confirmed that charge interactions were taking place between $\mathrm{Fe}$ in adjacent $\mathrm{Y}$ and $\mathrm{Z}$ sites.

The hyperfine parameters from previous Mössbauer studies on tourmaline are shown in Figure 2a. Site assignments of the doublets are typically made in millimeters per second relative to Fe metal following the conventions of Burns (1972) and Saegusa et al. (1979) in Table 1. To date, tetrahedral $\mathrm{Fe}^{3+}$ has not been reported in tourmaline; however, if it is present, it might be expected to have Mossbauer parameters similar to those of the tetrahedral $\mathrm{Fe}^{3+}$ in such silicates as clintonite (Annersten and Olesch 1978), ferriannite (Dyar and Burns 1986), hibonite (Burns and Burns 1984), schorlomite (Schwartz et al. 1980), and sillimanite (Rossman et al. 1982), which are all roughly in a range with $\delta=0.20$ $\mathrm{mm} / \mathrm{s}$ and $\Delta=0.50 \mathrm{~mm} / \mathrm{s}$.

More recent work (Pieczka and Kraczka 1997 and Pie- 
TABLE 3. Chemical composition

\begin{tabular}{|c|c|c|c|c|c|c|c|c|c|c|c|c|c|}
\hline & 1 & 2 & 3 & 4 & 5 & 6 & 7 & 8 & 9 & 10 & 11 & 12 & 13 \\
\hline $\mathrm{SiO}_{2}$ & 34.15 & 33.27 & 35.74 & 34.44 & 37.38 & 33.47 & 36.24 & 35.41 & 33.95 & 35.63 & 34.21 & 33.43 & 36.67 \\
\hline $\mathrm{Al}_{2} \mathrm{O}_{3}$ & 22.78 & 33.29 & 34.91 & 22.68 & 30.56 & 32.08 & 35.17 & 35.12 & 29.55 & 35.77 & 30.93 & 31.37 & 27.59 \\
\hline $\mathrm{TiO}_{2}$ & 1.52 & 0.71 & 0.50 & 0.97 & 0.03 & 0.48 & 0.23 & 0.35 & 0.39 & 0.03 & 1.63 & 0.57 & 0.45 \\
\hline $\mathrm{FeO}$ & 6.56 & 14.48 & 2.37 & 16.02 & 0.00 & 16.05 & 7.97 & 12.29 & 0.00 & 9.41 & 12.46 & 0.00 & 3.39 \\
\hline $\mathrm{Fe}_{2} \mathrm{O}_{3}$ & 8.56 & 0.00 & 4.30 & 0.00 & 0.07 & 1.14 & 0.00 & 0.00 & 1.34 & 1.85 & 1.20 & 20.03 & 4.60 \\
\hline $\mathrm{MgO}$ & 8.56 & 0.20 & 2.16 & 7.03 & 13.92 & 0.24 & 4.63 & 1.29 & 8.33 & 2.16 & 2.63 & 0.16 & 10.09 \\
\hline $\mathrm{MnO}$ & 0.01 & 1.10 & 0.18 & 0.03 & 0.00 & 0.16 & 0.01 & 0.09 & 0.00 & 0.01 & 0.04 & 0.11 & 0.05 \\
\hline $\mathrm{CaO}$ & 2.45 & 0.15 & 0.09 & 1.32 & 2.99 & 0.47 & 0.10 & 0.01 & 1.86 & 0.01 & 0.00 & 0.33 & 1.75 \\
\hline $\mathrm{Na}_{2} \mathrm{O}$ & 1.65 & 2.08 & 2.86 & 2.04 & 1.21 & 1.98 & 1.76 & 1.00 & 1.15 & 0.95 & 2.85 & 2.36 & 1.77 \\
\hline $\mathrm{K}_{2} \mathrm{O}$ & 0.00 & 0.00 & 0.00 & 0.02 & 0.03 & 0.04 & 0.02 & 0.01 & 0.13 & 0.02 & 0.05 & 0.06 & 0.06 \\
\hline $\mathrm{B}_{2} \mathrm{O}_{3}$ & 9.75 & 9.95 & 10.77 & 9.88 & 11.04 & 9.79 & 10.29 & 9.87 & 10.94 & 9.90 & 10.51 & 10.03 & 10.61 \\
\hline $\mathrm{ZnO}$ & & 0.22 & 0.04 & 0.03 & & & & & & & 0.07 & 0.00 & 0.00 \\
\hline $\mathrm{Cr}_{2} \mathrm{O}_{3}$ & 0.01 & 0.00 & 0.01 & 0.01 & 0.01 & 0.02 & 0.01 & 0.01 & 1.12 & 0.02 & 0.01 & 0.01 & 0.01 \\
\hline $\mathrm{Li}_{2} \mathrm{O}$ & 0.00 & 0.11 & 0.98 & 0.00 & 0.00 & 0.04 & 0.00 & 0.01 & 0.00 & 0.00 & 0.04 & 0.02 & 0.08 \\
\hline $\mathrm{V}_{2} \mathrm{O}_{3}$ & & & & & & & & & 7.03 & & 0.00 & 0.00 & 0.01 \\
\hline $\mathrm{F}$ & 0.45 & 0.30 & 0.20 & 0.11 & 1.22 & 1.08 & 0.15 & 0.20 & 0.54 & 0.08 & 0.36 & 1.52 & 0.67 \\
\hline $\mathrm{Cl}$ & & & & 0.00 & 0.00 & 0.01 & 0.00 & 0.00 & 0.00 & 0.00 & 0.00 & 0.01 & 0.01 \\
\hline $\mathrm{H}_{2} \mathrm{O}$ & 2.59 & 2.85 & 2.85 & 2.66 & 2.94 & 2.21 & 2.97 & 2.01 & 2.86 & 3.17 & 2.75 & 0.25 & 2.72 \\
\hline Total & 99.04 & 98.48 & 97.83 & 97.24 & 101.40 & 99.25 & 99.56 & 97.67 & 99.18 & 99.01 & 99.74 & 100.26 & 100.54 \\
\hline $\begin{array}{l}\text { No. of } \\
\text { analyses }\end{array}$ & 15 & 11 & 12 & 15 & 23 & 23 & 23 & 20 & 23 & 24 & 12 & 24 & 54 \\
\hline $\mathrm{Si}$ & 5.961 & 5.776 & 5.856 & 6.193 & 5.961 & 5.878 & 6.001 & 6.122 & 5.649 & 5.970 & 5.854 & 5.821 & 6.068 \\
\hline $\mathrm{Al}$ & 4.686 & 6.811 & 6.742 & 4.806 & 5.744 & 6.639 & 6.864 & 7.156 & 5.794 & 7.063 & 6.237 & 6.437 & 5.381 \\
\hline $\mathrm{Ti}$ & 0.200 & 0.093 & 0.062 & 0.131 & 0.004 & 0.063 & 0.029 & 0.046 & 0.049 & 0.004 & 0.210 & 0.075 & 0.056 \\
\hline $\mathrm{Fe}^{2+}$ & 0.957 & 2.102 & 0.325 & 2.409 & 0.000 & 2.356 & 1.104 & 1.777 & 0.000 & 1.318 & 1.782 & 0.000 & 0.469 \\
\hline $\mathrm{Fe}^{3+}$ & 1.124 & 0.000 & 0.530 & 0.000 & 0.008 & 0.150 & 0.000 & 0.000 & 0.168 & 0.233 & 0.155 & 2.624 & 0.573 \\
\hline $\mathrm{Mg}$ & 2.227 & 0.052 & 0.528 & 1.885 & 3.310 & 0.063 & 1.143 & 0.333 & 2.066 & 0.540 & 0.671 & 0.042 & 2.489 \\
\hline $\mathrm{Mn}$ & 0.001 & 0.162 & 0.025 & 0.005 & 0.000 & 0.024 & 0.001 & 0.013 & 0.000 & 0.001 & 0.006 & 0.016 & 0.007 \\
\hline $\mathrm{Ca}$ & 0.458 & 0.028 & 0.016 & 0.254 & 0.511 & 0.088 & 0.018 & 0.002 & 0.332 & 0.002 & 0.000 & 0.062 & 0.310 \\
\hline $\mathrm{Na}$ & 0.558 & 0.700 & 0.909 & 0.711 & 0.374 & 0.674 & 0.565 & 0.335 & 0.371 & 0.309 & 0.945 & 0.797 & 0.568 \\
\hline $\mathrm{K}$ & 0.000 & 0.000 & 0.000 & 0.005 & 0.006 & 0.009 & 0.004 & 0.002 & 0.028 & 0.004 & 0.011 & 0.013 & 0.013 \\
\hline$B$ & 2.938 & 2.980 & 3.046 & 3.065 & 3.039 & 2.967 & 2.941 & 2.946 & 3.140 & 2.864 & 3.104 & 3.015 & 3.031 \\
\hline $\mathrm{Zn}$ & 0.000 & 0.028 & 0.005 & 0.004 & 0.000 & 0.000 & 0.000 & 0.000 & 0.000 & 0.000 & 0.009 & 0.000 & 0.000 \\
\hline $\mathrm{Cr}$ & 0.001 & 0.000 & 0.001 & 0.001 & 0.001 & 0.003 & 0.001 & 0.001 & 0.147 & 0.003 & 0.001 & 0.001 & 0.001 \\
\hline $\mathrm{Li}$ & 0.001 & 0.078 & 0.643 & 0.001 & 0.003 & 0.025 & 0.001 & 0.009 & 0.001 & 0.000 & 0.029 & 0.016 & 0.055 \\
\hline $\mathrm{V}$ & 0.000 & 0.000 & 0.000 & 0.000 & 0.000 & 0.000 & 0.000 & 0.000 & 0.938 & 0.000 & 0.000 & 0.000 & 0.001 \\
\hline $\mathrm{F}$ & 0.246 & 0.332 & 0.913 & 0.063 & 0.613 & 0.602 & 0.080 & 0.109 & 0.282 & 0.042 & 0.193 & 0.836 & 0.351 \\
\hline $\mathrm{Cl}$ & 0.000 & 0.000 & 0.000 & 0.000 & 0.000 & 0.003 & 0.000 & 0.000 & 0.000 & 0.000 & 0.000 & 0.003 & 0.003 \\
\hline $\mathrm{H}$ & 3.015 & 3.300 & 3.109 & 3.191 & 3.127 & 2.583 & 3.280 & 2.314 & 3.169 & 3.543 & 3.139 & 0.285 & 3.002 \\
\hline
\end{tabular}

czka et al. 1997) suggests that both $\mathrm{Fe}^{3+}$ and $\mathrm{Fe}^{2+}$ occupy only the $\mathrm{Y}$ site in the structure and argues that previous assignment of $\mathrm{Fe}$ to the $\mathrm{Z}$ site just does not make sense crystallochemically. The multiple doublets previously attributed to $\mathrm{Fe}^{2+}$ are attributed to various combinations of nearest and next nearest neighbors, as follows from Pieczka et al. (1997) in Table 1.

These authors noted that the gradual decrease in quadrupole splitting (which is also accompanied by line broadening) is due to the decreasing contributions of ionic bonds in the Y octahedra when ions of higher valencies are present as next nearest neighbors. In this paper, both the Burns (1972) and the Pieczka et al. (1997) interpretations are considered.

\section{Methods}

\section{Sample selection and preparation}

Samples for this study were selected to include representatives from a wide variety of parageneses and compositions, including (1) specimens from compositional extremes already well studied by single-crystal X-ray refinements and wet chemistry, (2) samples from suspected compositional extremes selected from museum collec- tions and other sources, and (3) tourmaline from the petrologically well-constrained metamorphic, plutonic, and pegmatitic occurrences in western and southern Maine (Table 2). Whenever possible, we were careful to select samples for which mineral assemblage data were available.

For each sample, a total of approximately $700 \mathrm{mg}$ of tourmaline was prepared for analysis using magnetic separation and hand picking. A few small grains of each sample were set aside for X-ray diffraction and microprobe studies; the same grain mounts were used for both electron microprobe analysis (EPMA) and SIMS. In addition, 200-400 mg of tourmaline were prepared for $\mathrm{H}$ extraction, 100-200 mg for particle-induced gamma-ray emission (PIGE) for the interlaboratory comparison study (Dyar et al., in preparation), and approximately 200-500 mg for Mössbauer spectroscopy. Only pristine tourmaline grains were used for this study; any grains containing visible alteration, inclusions, zoning, or impurities were rejected.

\section{Electron microprobe}

Samples were analyzed for $\mathrm{Si}, \mathrm{Al}, \mathrm{Ti}, \mathrm{Mg}, \mathrm{Mn}, \mathrm{Ca}, \mathrm{Na}$, $\mathrm{K}, \mathrm{Zn}, \mathrm{Cr}, \mathrm{V}$, and $\mathrm{Cl}$ by EMPA at several institutions 
TABLE 3-Continued

\begin{tabular}{|c|c|c|c|c|c|c|c|c|c|c|c|c|c|c|}
\hline & 14 & 15 & 16 & 17 & 18 & 19 & 20 & 21 & 22 & 23 & 24 & 25 & 26 & 27 \\
\hline $\mathrm{SiO}_{2}$ & 36.11 & 37.52 & 37.69 & 36.37 & 36.42 & 35.53 & 36.65 & 36.39 & 35.58 & 36.13 & 36.30 & 36.64 & 36.38 & 35.04 \\
\hline $\mathrm{Al}_{2} \mathrm{O}_{3}$ & 26.41 & 31.87 & 28.93 & 31.91 & 31.55 & 29.31 & 32.64 & 32.90 & 34.29 & 33.59 & 34.09 & 32.84 & 33.39 & 33.82 \\
\hline $\mathrm{TiO}_{2}$ & 0.49 & 1.02 & 0.61 & 0.43 & 0.51 & 0.29 & 0.29 & 0.33 & 0.42 & 0.56 & 0.65 & 0.93 & 0.20 & 0.36 \\
\hline $\mathrm{FeO}$ & 4.24 & 0.46 & 0.00 & 5.45 & 5.58 & 10.56 & 6.51 & 5.74 & 10.71 & 5.94 & 7.35 & 5.14 & 7.80 & 12.62 \\
\hline $\mathrm{Fe}_{2} \mathrm{O}_{3}$ & 3.56 & 0.00 & 0.10 & 0.75 & 3.34 & 2.07 & 1.43 & 2.24 & 0.69 & 1.76 & 0.00 & 0.30 & 0.00 & 0.00 \\
\hline $\mathrm{MgO}^{3}$ & 11.08 & 11.28 & 14.62 & 7.23 & 6.80 & 5.83 & 6.78 & 6.67 & 3.16 & 5.54 & 5.21 & 7.32 & 5.26 & 1.70 \\
\hline $\mathrm{MnO}$ & 0.01 & 0.01 & 0.01 & 0.04 & 0.01 & 0.04 & 0.02 & 0.00 & 0.09 & 0.04 & 0.03 & 0.05 & 0.14 & 0.20 \\
\hline $\mathrm{CaO}$ & 2.74 & 0.46 & 3.29 & 0.30 & 0.45 & 0.84 & 0.40 & 0.59 & 0.22 & 0.84 & 0.15 & 0.42 & 0.09 & 0.13 \\
\hline $\mathrm{Na}_{2} \mathrm{O}$ & 1.25 & 2.23 & 1.08 & 2.33 & 2.03 & 2.12 & 1.94 & 1.88 & 1.79 & 1.17 & 1.71 & 1.93 & 2.12 & 1.76 \\
\hline $\mathrm{K}_{2} \mathrm{O}$ & 0.04 & 0.02 & 0.03 & 0.01 & 0.00 & 0.00 & 0.00 & 0.00 & 0.00 & 0.05 & 0.11 & 0.10 & 0.05 & 0.07 \\
\hline $\mathrm{B}_{2} \mathrm{O}_{3}$ & 10.09 & 10.75 & 11.91 & 10.38 & 10.60 & 10.35 & 10.78 & 10.66 & 10.87 & 10.96 & 10.59 & 11.31 & 11.07 & 10.28 \\
\hline $\mathrm{ZnO}$ & 0.00 & 0.00 & 0.00 & 0.02 & & & & & & & & & & \\
\hline $\mathrm{Cr}_{2} \mathrm{O}_{3}$ & 0.01 & 0.01 & 0.02 & 0.01 & 0.00 & 0.01 & 0.01 & 0.01 & 0.01 & 0.05 & 0.01 & 0.04 & 0.00 & 0.01 \\
\hline $\mathrm{Li}_{2} \mathrm{O}$ & 0.00 & 0.00 & 0.01 & 0.00 & 0.00 & 0.0014 & 0.00 & 0.00 & 0.01 & 0.00 & 0.00 & 0.03 & 0.03 & 0.09 \\
\hline $\mathrm{V}_{2} \mathrm{O}_{3}$ & 0.00 & 0.00 & 0.00 & & & & & & & 0.04 & 0.10 & 0.07 & 0.05 & 0.03 \\
\hline $\mathrm{F}$ & 1.06 & 0.24 & 1.23 & 0.24 & 0.08 & 0.03 & 0.01 & 0.17 & 0.33 & 0.11 & 0.20 & 0.58 & 0.53 & 0.51 \\
\hline $\mathrm{Cl}$ & 0.01 & 0.00 & 0.00 & 0.01 & & & & & 0.00 & 0.00 & 0.00 & 0.00 & 0.00 & 0.01 \\
\hline $\mathrm{H}_{2} \mathrm{O}$ & 2.62 & 3.00 & 2.56 & 2.39 & 2.46 & 2.94 & 3.03 & 3.11 & 2.92 & 2.80 & 2.75 & 2.44 & 2.94 & 2.82 \\
\hline Total & 99.73 & 98.88 & 102.09 & 97.87 & 99.83 & 99.91 & 100.49 & 100.69 & 101.09 & 99.58 & 99.25 & 100.14 & 100.05 & 99.46 \\
\hline \multicolumn{15}{|l|}{ No. of } \\
\hline analyses & 24 & 24 & 6 & 1 & 5 & 6 & 6 & 6 & 6 & 5 & 5 & 5 & 4 & 23 \\
\hline $\mathrm{Si}$ & 6.068 & 6.089 & 5.982 & 6.132 & 6.064 & 6.025 & 6.013 & 5.956 & 5.888 & 5.964 & 6.027 & 6.003 & 5.994 & 5.948 \\
\hline $\mathrm{Al}$ & 5.230 & 6.095 & 5.412 & 6.341 & 6.191 & 5.858 & 6.311 & 6.346 & 6.687 & 6.535 & 6.671 & 6.340 & 6.483 & 6.765 \\
\hline $\mathrm{Ti}$ & 0.062 & 0.124 & 0.073 & 0.055 & 0.064 & 0.037 & 0.036 & 0.041 & 0.052 & 0.070 & 0.081 & 0.115 & 0.025 & 0.046 \\
\hline $\mathrm{Fe}^{2+}$ & 0.596 & 0.062 & 0.000 & 0.768 & 0.776 & 1.497 & 0.893 & 0.786 & 1.482 & 0.820 & 1.020 & 0.704 & 1.075 & 1.791 \\
\hline $\mathrm{Fe}^{3+}$ & 0.450 & 0.000 & 0.012 & 0.095 & 0.418 & 0.264 & 0.177 & 0.276 & 0.086 & 0.218 & 0.000 & 0.037 & 0.000 & 0.000 \\
\hline $\mathrm{Mg}$ & 2.776 & 2.729 & 3.460 & 1.817 & 1.688 & 1.474 & 1.658 & 1.627 & 0.780 & 1.363 & 1.290 & 1.788 & 1.292 & 0.430 \\
\hline $\mathrm{Mn}$ & 0.001 & 0.001 & 0.001 & 0.006 & 0.001 & 0.006 & 0.003 & 0.000 & 0.013 & 0.006 & 0.004 & 0.007 & 0.020 & 0.029 \\
\hline $\mathrm{Ca}$ & 0.493 & 0.080 & 0.559 & 0.054 & 0.080 & 0.153 & 0.070 & 0.103 & 0.039 & 0.149 & 0.027 & 0.074 & 0.016 & 0.024 \\
\hline $\mathrm{Na}$ & 0.407 & 0.702 & 0.332 & 0.762 & 0.655 & 0.697 & 0.617 & 0.597 & 0.574 & 0.374 & 0.550 & 0.613 & 0.677 & 0.579 \\
\hline $\mathrm{K}$ & 0.009 & 0.004 & 0.006 & 0.002 & 0.000 & 0.000 & 0.000 & 0.000 & 0.000 & 0.011 & 0.023 & 0.021 & 0.011 & 0.015 \\
\hline$B$ & 2.928 & 3.012 & 3.263 & 3.021 & 3.046 & 3.028 & 3.051 & 3.011 & 3.105 & 3.122 & 3.034 & 3.198 & 3.147 & 3.012 \\
\hline $\mathrm{Zn}$ & 0.000 & 0.000 & 0.000 & 0.002 & 0.000 & 0.000 & 0.000 & 0.000 & 0.000 & 0.000 & 0.000 & 0.000 & 0.000 & 0.000 \\
\hline $\mathrm{Cr}$ & 0.001 & 0.001 & 0.003 & 0.001 & 0.000 & 0.001 & 0.001 & 0.001 & 0.001 & 0.007 & 0.001 & 0.005 & 0.000 & 0.001 \\
\hline $\mathrm{Li}$ & 0.002 & 0.002 & 0.004 & 0.002 & 0.001 & 0.001 & 0.001 & 0.001 & 0.006 & 0.003 & 0.002 & 0.017 & 0.018 & 0.064 \\
\hline V & 0.000 & 0.000 & 0.000 & 0.000 & 0.000 & 0.000 & 0.000 & 0.000 & 0.000 & 0.005 & 0.013 & 0.009 & 0.007 & 0.004 \\
\hline $\mathrm{F}$ & 0.564 & 0.123 & 0.618 & 0.127 & 0.044 & 0.013 & 0.004 & 0.087 & 0.171 & 0.057 & 0.105 & 0.300 & 0.276 & 0.274 \\
\hline $\mathrm{Cl}$ & 0.003 & 0.000 & 0.000 & 0.003 & 0.000 & 0.000 & 0.000 & 0.000 & 0.000 & 0.000 & 0.000 & 0.000 & 0.000 & 0.003 \\
\hline $\mathrm{H}$ & 2.937 & 3.247 & 2.710 & 2.688 & 2.732 & 3.326 & 3.316 & 3.395 & 3.223 & 3.083 & 3.046 & 2.666 & 3.231 & 3.193 \\
\hline
\end{tabular}

participating in a related project on the interlaboratory reproducibility of tourmaline analyses. Analysts and laboratories included Jeremy Delaney at Rutgers University, James McGee at the U.S.G.S. in Reston, Anne McGuire of the Texas Center for Superconductivity at the University of Houston, Virginia Sisson at Rice University, and Michael Wise at the National Museum of Natural History. Additional analyses were also taken from the original published work on samples that had been previously studied. Analytical instrumentation, experimental conditions, and standards are described elsewhere (Dyar et al., in preparation). EMPA scans at the University of Houston were used to screen for zoning in tourmaline; zoned samples were eliminated from this study. An average of 22 analyses from four different laboratories was obtained for each sample, and analytical errors are estimated to be $\pm 0.5-2 \%$ for major elements and $\pm 10-20 \%$ for minor elements. B was analyzed by PIGE at the University of Kentucky, by prompt gamma neutron activation analysis (PGNAA) at the NIST, and by EMPA by all participants in this study except Michael Wise; however, only the SIMS B results, which are all internally consistent and which were made at the same scale on the same grains as the EMPA analyses, are used here. Comparisons be- tween the electron microprobe B data and other techniques are presented elsewhere (Dyar et al., in preparation).

\section{H extraction}

Samples were analyzed for H contents by Darby Dyar in the Stable Isotope Laboratory at Southern Methodist University. $\mathrm{H}$ contents were determined using a method for volumetric measurement of $\mathrm{H}_{2} \mathrm{O}$ vapor extracted from silicates (Bigeleisen et al. 1952 and Holdaway et al. 1986). In general, clean mineral separates were degassed under vacuum for at least $8 \mathrm{~h}$ at $50-85{ }^{\circ} \mathrm{C}$ to drive off absorbed atmospheric moisture. Samples were then fused in an induction furnace to liberate structural $\mathrm{H}_{2} \mathrm{O}$. Distillation processes involving transfer of evolved gases through a series of liquid nitrogen and methanol-dry ice slush traps were used to separate $\mathrm{H}_{2} \mathrm{O}$ molecules effectively from other condensable and non-condensable gases. $\mathrm{H}_{2} \mathrm{O}$ vapor was then passed over a hot $\left(>750{ }^{\circ} \mathrm{C}\right)$ uranium furnace to liberate free $\mathrm{H}^{+}$. A mercury-piston Toepler pump was used to collect $\mathrm{H}$ vapor in a volumetrically calibrated reservoir for yield measurement. 
TABLE 3-Continued

\begin{tabular}{|c|c|c|c|c|c|c|c|c|c|c|c|c|c|}
\hline & 28 & 29 & 30 & 31 & 32 & 33 & 34 & 35 & 36 & 37 & 38 & 39 & 40 \\
\hline $\mathrm{SiO}_{2}$ & 34.51 & 35.15 & 36.53 & 36.53 & 36.56 & 34.71 & 35.33 & 35.37 & 35.08 & 36.04 & 35.03 & 35.27 & 37.66 \\
\hline $\mathrm{Al}_{2} \mathrm{O}_{3}$ & 32.45 & 32.56 & 32.27 & 37.11 & 36.73 & 34.03 & 34.21 & 33.93 & 34.53 & 38.13 & 34.87 & 33.96 & 40.12 \\
\hline $\mathrm{TiO}_{2}$ & 1.03 & 1.00 & 0.26 & 0.01 & 0.02 & 0.12 & 0.32 & 0.66 & 0.65 & 0.17 & 0.08 & 0.11 & 0.04 \\
\hline $\mathrm{FeO}$ & 13.61 & 9.80 & 2.27 & 6.98 & 8.76 & 11.62 & 10.56 & 7.78 & 11.36 & 6.87 & 12.61 & 12.38 & 2.47 \\
\hline $\mathrm{Fe}_{2} \mathrm{O}_{3}$ & 0.00 & 0.57 & 0.31 & 0.00 & 0.00 & 1.36 & 0.55 & 1.18 & 0.00 & 0.00 & 0.00 & 0.00 & 0.00 \\
\hline $\mathrm{MgO}$ & 1.96 & 4.00 & 10.42 & 0.00 & 0.66 & 1.59 & 3.10 & 4.83 & 1.47 & 0.53 & 1.54 & 2.04 & 0.11 \\
\hline $\mathrm{MnO}$ & 0.21 & 0.10 & 0.01 & 0.29 & 0.14 & 0.14 & 0.11 & 0.04 & 0.24 & 0.23 & 0.23 & 0.23 & 0.36 \\
\hline $\mathrm{CaO}$ & 0.26 & 0.20 & 2.06 & 0.09 & 0.05 & 0.04 & 0.19 & 0.53 & 0.05 & 0.12 & 0.04 & 0.05 & 0.38 \\
\hline $\mathrm{Na}_{2} \mathrm{O}$ & 1.98 & 2.05 & 1.45 & 2.41 & 2.23 & 1.79 & 1.84 & 1.87 & 1.90 & 2.36 & 1.60 & 1.74 & 1.93 \\
\hline $\mathrm{K}_{2} \mathrm{O}$ & 0.05 & 0.03 & 0.05 & 0.03 & 0.03 & 0.03 & 0.03 & 0.04 & 0.03 & 0.03 & 0.01 & 0.01 & 0.01 \\
\hline $\mathrm{B}_{2} \mathrm{O}_{3}$ & 10.12 & 10.31 & 10.69 & 10.83 & 10.68 & 9.90 & 10.27 & 10.18 & 10.59 & 10.77 & 10.35 & 10.23 & 11.91 \\
\hline $\mathrm{ZnO}$ & & 0.05 & & & & & & & & 0.04 & & & 0.07 \\
\hline $\mathrm{Cr}_{2} \mathrm{O}_{3}$ & 0.01 & 0.01 & 0.05 & 0.00 & 0.01 & 0.01 & 0.01 & 0.01 & 0.02 & 0.01 & 0.00 & 0.01 & 0.01 \\
\hline $\mathrm{Li}_{2} \mathrm{O}$ & 0.09 & 0.01 & 0.02 & 1.31 & 1.04 & 0.05 & 0.03 & 0.01 & 0.36 & 1.41 & 0.18 & 0.12 & 2.10 \\
\hline $\mathrm{V}_{2} \mathrm{O}_{3}$ & & & 0.06 & & & & & & & & & & \\
\hline $\mathrm{F}^{23}$ & 0.56 & 0.56 & 0.20 & 1.37 & 1.13 & 0.31 & 0.20 & 0.18 & 0.71 & 1.32 & 0.34 & 0.53 & 1.26 \\
\hline $\mathrm{Cl}$ & 0.00 & 0.00 & 0.00 & 0.00 & 0.00 & 0.00 & 0.00 & 0.00 & 0.00 & 0.00 & & & 0.00 \\
\hline $\mathrm{H}_{2} \mathrm{O}$ & 2.77 & 2.81 & 2.75 & 2.69 & 2.93 & 3.13 & 2.94 & 2.90 & 2.78 & 2.68 & 3.02 & 2.89 & 2.94 \\
\hline Total & 99.60 & 99.22 & 99.40 & 99.65 & 100.97 & 98.82 & 99.69 & 99.50 & 99.77 & 100.71 & 99.90 & 99.57 & 101.38 \\
\hline $\begin{array}{l}\text { No. of } \\
\text { analyses }\end{array}$ & 23 & 1 & 21 & 23 & 39 & 22 & 23 & 23 & 23 & 23 & 7 & 7 & 7 \\
\hline $\mathrm{Si}$ & 5.906 & 5.938 & 5.983 & 5.991 & 5.963 & 5.914 & 5.934 & 5.910 & 5.893 & 5.859 & 5.895 & 5.965 & 5.895 \\
\hline $\mathrm{Al}$ & 6.545 & 6.483 & 6.229 & 7.173 & 7.061 & 6.833 & 6.772 & 6.681 & 6.836 & 7.305 & 6.915 & 6.769 & 7.401 \\
\hline $\mathrm{Ti}$ & 0.133 & 0.127 & 0.032 & 0.001 & 0.002 & 0.015 & 0.040 & 0.083 & 0.082 & 0.021 & 0.010 & 0.014 & 0.005 \\
\hline $\mathrm{Fe}^{2+}$ & 1.948 & 1.385 & 0.311 & 0.957 & 1.195 & 1.655 & 1.483 & 1.087 & 1.596 & 0.934 & 1.774 & 1.751 & 0.323 \\
\hline $\mathrm{Fe}^{3+}$ & 0.000 & 0.073 & 0.038 & 0.000 & 0.000 & 0.174 & 0.070 & 0.148 & 0.000 & 0.000 & 0.000 & 0.000 & 0.000 \\
\hline $\mathrm{Mg}$ & 0.500 & 1.007 & 2.544 & 0.000 & 0.160 & 0.404 & 0.776 & 1.203 & 0.368 & 0.128 & 0.386 & 0.514 & 0.026 \\
\hline $\mathrm{Mn}$ & 0.030 & 0.014 & 0.001 & 0.040 & 0.019 & 0.020 & 0.016 & 0.006 & 0.034 & 0.032 & 0.033 & 0.033 & 0.048 \\
\hline $\mathrm{Ca}$ & 0.048 & 0.036 & 0.361 & 0.016 & 0.009 & 0.007 & 0.034 & 0.095 & 0.009 & 0.021 & 0.007 & 0.009 & 0.064 \\
\hline $\mathrm{Na}$ & 0.657 & 0.671 & 0.460 & 0.766 & 0.705 & 0.591 & 0.599 & 0.606 & 0.619 & 0.744 & 0.522 & 0.571 & 0.586 \\
\hline $\mathrm{K}$ & 0.011 & 0.006 & 0.010 & 0.006 & 0.006 & 0.007 & 0.006 & 0.009 & 0.006 & 0.006 & 0.002 & 0.002 & 0.002 \\
\hline$B$ & 2.989 & 3.005 & 3.021 & 3.066 & 3.007 & 2.910 & 2.977 & 2.936 & 3.071 & 3.022 & 3.006 & 2.986 & 3.218 \\
\hline $\mathrm{Zn}$ & 0.000 & 0.006 & 0.000 & 0.000 & 0.000 & 0.000 & 0.000 & 0.000 & 0.000 & 0.005 & 0.000 & 0.000 & 0.008 \\
\hline $\mathrm{Cr}$ & 0.001 & 0.001 & 0.006 & 0.000 & 0.001 & 0.001 & 0.001 & 0.001 & 0.003 & 0.001 & 0.000 & 0.001 & 0.001 \\
\hline $\mathrm{Li}$ & 0.058 & 0.010 & 0.014 & 0.865 & 0.682 & 0.035 & 0.019 & 0.006 & 0.240 & 0.922 & 0.121 & 0.085 & 1.325 \\
\hline $\mathrm{V}$ & 0.000 & 0.000 & 0.008 & 0.000 & 0.000 & 0.000 & 0.000 & 0.000 & 0.000 & 0.000 & 0.000 & 0.000 & 0.000 \\
\hline $\mathrm{F}$ & 0.301 & 0.299 & 0.104 & 0.709 & 0.583 & 0.168 & 0.105 & 0.093 & 0.377 & 0.679 & 0.181 & 0.283 & 0.624 \\
\hline $\mathrm{Cl}$ & 0.000 & 0.000 & 0.000 & 0.000 & 0.000 & 0.000 & 0.000 & 0.000 & 0.000 & 0.000 & 0.000 & 0.000 & 0.000 \\
\hline $\mathrm{H}$ & 3.162 & 3.166 & 3.004 & 2.943 & 3.188 & 3.557 & 3.294 & 3.232 & 3.115 & 2.906 & 3.390 & 3.260 & 3.069 \\
\hline
\end{tabular}

\section{Secondary ion mass spectrometry}

Analyses of B and Li were performed by Grant Fowler and Michael Wiedenbeck at the University of New Mexico/Sandia National Laboratory SIMS facility using a Cameca IMS 4f ion microprobe and the same grain mounts used for electron microprobe study. A primary O-plasma accelerated to $12.5 \mathrm{KV}$ was used, and the $20 \mathrm{nA}$ beam was focused to a diameter of $\sim 25 \mu \mathrm{m}$. Secondary ions were accelerated using a nominally $4.5 \mathrm{kV}$ potential to which a $50 \mathrm{~V}$ offset energy filter was applied using a 25 $\mathrm{V}$ full width energy window. The secondary ion beam was mass separated using a $90^{\circ}$ sector magnet operated in low mass resolution mode $(\mathrm{M} / \mathrm{dM} \cong 360)$. Ions were detected using an ETP electron multiplier operated in pulse counting mode; count rates were kept below $\sim 20$ MHZ in order to minimize the impact of detector deadtime corrections.

Li and B concentration analyses involved magnet peakstepping that included four mass stations: 6.5 background $(2 \mathrm{~s}),{ }^{7} \mathrm{Li}(2 \mathrm{~s}),{ }^{11} \mathrm{~B}(3 \mathrm{~s})$ and ${ }^{30} \mathrm{Si}(2 \mathrm{~s})$; all data were normalized to the observed ${ }^{30} \mathrm{Si}$ intensity. Mineral standards analyzed by nuclear techniques and wet chemistry were used (Francis et al. 1994). All samples were ana- lyzed twice and the results were averaged to obtain the results given in Table 3. Standard deviations were generally less than $0.02 \mathrm{wt} \% \mathrm{~B}_{2} \mathrm{O}_{3}$ and $\mathrm{Li}_{2} \mathrm{O}$. Additional information on the method is given in Hervig (1996).

\section{Mössbauer spectroscopy}

Mössbauer mounts were prepared by mixing sucrose with sample under acetone. Where sufficient material was available (approximately half the samples), mounts were prepared to satisfy the ideal absorber thickness approximation of Long et al. (1983) based on the tourmaline compositions. In all other cases, at least $100 \mathrm{mg}$ of sample (usually enough to yield $2-5 \mathrm{mg} \mathrm{Fe} / \mathrm{cm}^{2}$ in the holder, but in cases of extremely low Fe samples, as little as 0.02 $\mathrm{mg} \mathrm{Fe} / \mathrm{cm}^{2}$ ) were used. Of course, the low Fe samples yielded fits with inferior statistics [see columns labeled Mis (\%) and Un (\%) in Table 4], but otherwise no significant differences were noted between the two populations of samples.

Room-temperature Mössbauer studies to determine $\mathrm{Fe}^{2+}$ and $\mathrm{Fe}^{3+}$ content were done in the Mineral Spectroscopy Laboratory at West Chester University and formerly at the University of Oregon. A source of 50-20 
TABLE 3-Continued

\begin{tabular}{|c|c|c|c|c|c|c|c|c|c|c|c|c|c|c|}
\hline & 41 & 42 & 43 & 44 & 45 & 46 & 47 & 48 & 49 & 50 & 51 & 52 & 53 & 54 \\
\hline $\mathrm{SiO}_{2}$ & 36.04 & 36.57 & 36.34 & 36.28 & 37.77 & 37.99 & 38.36 & 36.58 & 35.46 & 35.47 & 35.45 & 34.11 & 37.47 & 35.67 \\
\hline $\mathrm{Al}_{2} \mathrm{O}_{3}$ & 33.84 & 36.72 & 36.94 & 37.66 & 43.90 & 42.84 & 42.27 & 38.56 & 33.71 & 33.75 & 33.83 & 35.96 & 34.33 & 36.90 \\
\hline $\mathrm{TiO}_{2}$ & 0.77 & 0.05 & 0.03 & 0.02 & 0.02 & 0.02 & 0.02 & 0.04 & 0.12 & 0.15 & 0.31 & 0.39 & 0.09 & 0.00 \\
\hline $\mathrm{FeO}$ & 8.01 & 6.74 & 7.71 & 6.83 & 0.01 & 0.00 & 0.00 & 4.94 & 10.81 & 12.10 & 10.96 & 8.52 & 2.46 & 1.63 \\
\hline $\mathrm{Fe}_{2} \mathrm{O}_{3}$ & 0.00 & 0.00 & 0.00 & 0.00 & 0.06 & 0.04 & 0.06 & 0.00 & 1.04 & 0.78 & 0.57 & 0.50 & 0.96 & 0.29 \\
\hline $\mathrm{MgO}$ & 5.15 & 0.01 & 0.00 & 0.02 & 0.01 & 0.00 & 0.00 & 0.01 & 2.49 & 1.61 & 2.71 & 3.50 & 9.16 & 9.34 \\
\hline $\mathrm{MnO}$ & 0.21 & 0.37 & 0.23 & 0.49 & 0.44 & 0.41 & 0.27 & 0.63 & 0.11 & 0.12 & 0.13 & 0.03 & 0.03 & 0.02 \\
\hline $\mathrm{CaO}$ & 0.20 & 0.10 & 0.09 & 0.09 & 0.02 & 0.06 & 0.36 & 0.25 & 0.13 & 0.09 & 0.12 & 0.57 & 1.18 & 1.66 \\
\hline $\mathrm{Na}_{2} \mathrm{O}$ & 1.97 & 2.58 & 2.03 & 2.17 & 1.51 & 1.58 & 1.59 & 2.38 & 1.71 & 1.66 & 1.84 & 2.25 & 2.04 & 2.15 \\
\hline $\mathrm{K}_{2} \mathrm{O}$ & 0.01 & 0.03 & 0.01 & 0.01 & 0.01 & 0.02 & 0.02 & 0.02 & 0.07 & 0.03 & 0.03 & 0.19 & 0.02 & 0.01 \\
\hline $\mathrm{B}_{2} \mathrm{O}_{3}$ & 10.86 & 10.82 & 10.74 & 10.86 & 11.54 & 12.16 & 12.09 & 11.00 & 10.71 & 10.46 & 10.47 & 10.89 & 11.42 & 11.88 \\
\hline $\mathrm{ZnO}$ & & 0.75 & & & 0.27 & 0.18 & 0.07 & 0.49 & 0.05 & 0.12 & 0.68 & 0.06 & 0.01 & 0.01 \\
\hline $\mathrm{Cr}_{2} \mathrm{O}_{3}$ & 0.01 & 0.02 & 0.01 & 0.00 & 0.01 & 0.01 & 0.01 & 0.00 & 0.01 & 0.00 & 0.01 & 0.03 & & \\
\hline $\mathrm{Li}_{2} \mathrm{O}$ & 0.03 & 1.60 & 1.28 & 1.39 & 1.54 & 1.86 & 2.35 & 1.67 & 0.01 & 0.02 & 0.01 & 0.04 & 0.00 & 0.00 \\
\hline $\mathrm{V}_{2} \mathrm{O}_{3}$ & & & & & & & & & 0.01 & & & 0.05 & & \\
\hline $\mathrm{F}^{23}$ & 0.28 & 1.51 & 1.08 & 1.13 & 0.57 & 0.95 & 1.11 & 1.54 & 0.38 & 0.33 & 0.26 & 0.54 & 0.00 & 0.00 \\
\hline $\mathrm{Cl}$ & & 0.00 & & & 0.00 & 0.00 & 0.00 & 0.00 & 0.01 & 0.00 & 0.00 & 0.00 & & 0.00 \\
\hline $\mathrm{H}_{2} \mathrm{O}$ & 2.80 & 2.77 & 2.68 & 2.88 & 3.41 & 3.66 & 3.71 & 2.89 & 3.06 & 3.02 & 3.03 & 3.13 & 3.21 & 3.19 \\
\hline Total & 100.18 & 100.64 & 99.17 & 99.82 & 101.09 & 101.78 & 102.28 & 101.00 & 99.90 & 99.70 & 100.42 & 100.75 & 102.38 & 102.74 \\
\hline $\begin{array}{l}\text { No. of } \\
\text { analyses }\end{array}$ & 7 & 1 & 7 & 6 & 23 & 23 & 23 & 7 & 2 & 2 & 2 & 1 & 1 & 1 \\
\hline $\mathrm{Si}$ & 5.952 & 5.962 & 5.999 & 5.925 & 5.837 & 5.807 & 5.832 & 5.878 & 5.936 & 5.981 & 5.933 & 5.627 & 5.923 & 5.600 \\
\hline $\mathrm{Al}$ & 6.587 & 7.055 & 7.187 & 7.249 & 7.996 & 7.717 & 7.573 & 7.302 & 6.650 & 6.707 & 6.673 & 6.991 & 6.395 & 6.827 \\
\hline $\mathrm{Ti}$ & 0.096 & 0.006 & 0.004 & 0.002 & 0.002 & 0.002 & 0.002 & 0.005 & 0.015 & 0.019 & 0.039 & 0.048 & 0.011 & 0.000 \\
\hline $\mathrm{Fe}^{2+}$ & 1.106 & 0.919 & 1.064 & 0.933 & 0.002 & 0.000 & 0.000 & 0.664 & 1.513 & 1.706 & 1.534 & 1.176 & 0.325 & 0.213 \\
\hline $\mathrm{Fe}^{3+}$ & 0.000 & 0.000 & 0.000 & 0.000 & 0.007 & 0.005 & 0.006 & 0.000 & 0.132 & 0.099 & 0.072 & 0.062 & 0.114 & 0.035 \\
\hline $\mathrm{Mg}$ & 1.268 & 0.002 & 0.000 & 0.005 & 0.002 & 0.000 & 0.000 & 0.002 & 0.621 & 0.405 & 0.676 & 0.861 & 2.158 & 2.186 \\
\hline $\mathrm{Mn}$ & 0.029 & 0.051 & 0.032 & 0.068 & 0.058 & 0.053 & 0.035 & 0.086 & 0.016 & 0.017 & 0.018 & 0.004 & 0.004 & 0.003 \\
\hline $\mathrm{Ca}$ & 0.035 & 0.017 & 0.016 & 0.016 & 0.003 & 0.010 & 0.059 & 0.043 & 0.023 & 0.016 & 0.022 & 0.101 & 0.200 & 0.279 \\
\hline $\mathrm{Na}$ & 0.631 & 0.815 & 0.650 & 0.687 & 0.452 & 0.468 & 0.469 & 0.741 & 0.555 & 0.543 & 0.597 & 0.720 & 0.625 & 0.654 \\
\hline $\mathrm{K}$ & 0.002 & 0.006 & 0.002 & 0.002 & 0.002 & 0.004 & 0.004 & 0.004 & 0.015 & 0.006 & 0.006 & 0.040 & 0.004 & 0.002 \\
\hline$B$ & 3.094 & 3.045 & 3.061 & 3.062 & 3.077 & 3.207 & 3.173 & 3.052 & 3.095 & 3.044 & 3.024 & 3.100 & 3.116 & 3.218 \\
\hline $\mathrm{Zn}$ & 0.000 & 0.090 & 0.000 & 0.000 & 0.031 & 0.020 & 0.008 & 0.058 & 0.006 & 0.015 & 0.084 & 0.007 & 0.001 & 0.001 \\
\hline $\mathrm{Cr}$ & 0.001 & 0.003 & 0.001 & 0.000 & 0.001 & 0.001 & 0.001 & 0.000 & 0.001 & 0.000 & 0.001 & 0.004 & 0.000 & 0.000 \\
\hline $\mathrm{Li}$ & 0.020 & 1.048 & 0.851 & 0.911 & 0.958 & 1.145 & 1.435 & 1.078 & 0.007 & 0.011 & 0.007 & 0.025 & 0.002 & 0.002 \\
\hline $\mathrm{V}$ & 0.000 & 0.000 & 0.000 & 0.000 & 0.000 & 0.000 & 0.000 & 0.000 & 0.001 & 0.000 & 0.000 & 0.007 & 0.000 & 0.000 \\
\hline $\mathrm{F}$ & 0.146 & 0.778 & 0.564 & 0.584 & 0.279 & 0.459 & 0.534 & 0.782 & 0.201 & 0.176 & 0.138 & 0.282 & 0.000 & 0.000 \\
\hline $\mathrm{Cl}$ & 0.000 & 0.000 & 0.000 & 0.000 & 0.000 & 0.000 & 0.000 & 0.000 & 0.003 & 0.000 & 0.000 & 0.000 & 0.000 & 0.000 \\
\hline $\mathrm{H}$ & 3.084 & 3.012 & 2.951 & 3.132 & 3.515 & 3.726 & 3.757 & 3.097 & 3.417 & 3.397 & 3.383 & 3.444 & 3.384 & 3.335 \\
\hline
\end{tabular}

$\mathrm{mCi}{ }^{57} \mathrm{Co}$ in $\mathrm{Rh}$ was used on an Austin Science Associates constant acceleration spectrometer. Experiment times for the individual measurements averaged two to three days per sample. Results were calibrated against an $\alpha$-Fe foil of $6 \mu \mathrm{m}$ thickness and $99 \%$ purity. Spectra were fit using an unpublished version of the program STONE modified to run on IBM and compatible personal computers. The program uses a nonlinear regression procedure with a facility for constraining any set of parameters or linear combination of parameters. Lorentzian line shapes were used for resolving peaks, as there was no statistical justification for the addition of a Gaussian component to the peak shapes. Fitting procedures in general followed those described in Dyar et al. (1989) and McGuire et al. (1989). Fitting models were used to produce a statistical best fit for each spectrum using the $\chi^{2}$ and Misfit parameters (Dyar 1984), and are discussed in detail by Taylor (1996). Errors are estimated at $\pm 3 \%$ for doublet areas, and \pm 0.02 $\mathrm{mm} / \mathrm{s}$ for peak width $(\Gamma)$, isomer shift $(\delta)$, and quadrupole splitting $(\Delta)$.

\section{Mössbauer data}

\section{Results}

Seven different Mössbauer doublets were observed in various combinations for samples in this suite (Fig. 2b).
Averages for each of these groups are given at the bottom of Table 4.

$\mathbf{F e}^{2+}$ doublets. In 39 of the $47 \mathrm{Fe}^{2+}$-bearing samples, the two most prominent doublets correspond to $\mathrm{Fe}^{2+}$ with $\delta=1.09 \mathrm{~mm} / \mathrm{s}$ and $\Delta=$ approximately 2.47 and 2.28 $\mathrm{mm} / \mathrm{s}$. In all of these cases, simpler models using either (1) only one $\mathrm{Fe}^{2+}$ doublet with a starting $\Delta$ value of 2.3 $\mathrm{mm} / \mathrm{s}$ or (2) two doublets with $\Delta$ estimated at 2.47 and $1.56 \mathrm{~mm} / \mathrm{s}$ as found by Burns (1972) were attempted, but it proved impossible to obtain unconstrained, converged fits with those models for these spectra. This occurs because the $\mathrm{Fe}^{2+}$ doublets with the larger quadrupole splittings are much more intense than the third $\mathrm{Fe}^{2+}$ doublet. In 7 of those 47 samples, the model with doublets at $\Delta$ $=2.47$ and $1.56 \mathrm{~mm} / \mathrm{s}$ was successfully used, and in the remaining one spectrum only one $\mathrm{Fe}^{2+}$ doublet was found. This result is consistent with the observation by Pieckza et al. (1997) that the larger $\Delta \mathrm{Fe}^{2+}$ doublets are those with the sharpest, most intense peaks.

The two $\mathrm{Fe}^{2+}$ doublets with the highest $\Delta$ values both lie around the average for ${ }^{[\mathrm{Y}]} \mathrm{Fe}^{2+}$ assigned by previous workers (see Figs. $2 \mathrm{a}$ and $2 \mathrm{~b}$ ). Thus, we interpret the two $\mathrm{Fe}^{2+}$ doublets with the highest $\Delta$ values to represent $\mathrm{Fe}^{2+}$ in $\mathrm{Y}$ sites with different nearest neighbor coordination 
TABLE 4. Mössbauer results

\begin{tabular}{|c|c|c|c|c|c|c|c|c|c|c|c|c|}
\hline \multirow{2}{*}{$\begin{array}{l}\text { Sample } \\
\text { number }\end{array}$} & \multicolumn{4}{|c|}{$\mathrm{Fe}^{3+}$ in $\mathrm{Y} ?$ or $\mathrm{Si}$} & \multicolumn{4}{|c|}{$\mathrm{Fe}^{3+}$ in octahedra } & \multicolumn{4}{|c|}{$\mathrm{Fe}^{3+} \mathrm{Fe}^{2+} \mathrm{ED}$} \\
\hline & $\delta$ & $\Delta$ & $\Gamma$ & Area & $\delta$ & $\Delta$ & $\Gamma$ & Area & $\delta$ & $\Delta$ & $\Gamma$ & Area \\
\hline 1 & & & & & 0.40 & 0.74 & 0.40 & 54 & & & & \\
\hline 2 & & & & & & & & & & & & \\
\hline 3 & & & & & 0.40 & 0.77 & 0.40 & 62 & & & & \\
\hline 4 & & & & & & & & & & & & \\
\hline 5 & 0.18 & 0.46 & 0.26 & 100 & & & & & & & & \\
\hline 6 & 0.43 & 0.48 & 0.25 & 6 & & & & & & & & \\
\hline 7 & & & & & & & & & & & & \\
\hline 8 & & & & & & & & & & & & \\
\hline 9 & 0.13 & 0.47 & 0.45 & 100 & & & & & & & & \\
\hline 10 & & & & & 0.49 & 0.77 & 0.35 & 15 & & & & \\
\hline 11 & & & & & & & & & 0.76 & 1.02 & 0.58 & 16 \\
\hline 12 & & & & & 0.37 & 1.24 & 0.33 & 70 & & & & \\
\hline & & & & & 0.35 & 0.90 & 0.31 & 30 & & & & \\
\hline 13 & & & & & 0.39 & 0.78 & 0.50 & 55 & & & & \\
\hline 14 & & & & & 0.38 & 0.78 & 0.45 & 43 & & & & \\
\hline 15 & & & & & & & & & & & & \\
\hline 16 & 0.15 & 0.59 & 0.20 & 100 & & & & & & & & \\
\hline 17 & & & & & 0.45 & 0.73 & 0.73 & 11 & & & & \\
\hline 18 & & & & & 0.45 & 0.75 & 0.47 & 28 & 0.82 & 1.30 & 0.54 & 14 \\
\hline 19 & & & & & 0.41 & 0.73 & 0.60 & 12 & 0.79 & 1.08 & 0.50 & 6 \\
\hline 20 & & & & & 0.45 & 0.77 & 0.56 & 14 & 0.75 & 1.38 & 0.46 & 5 \\
\hline 21 & & & & & 0.45 & 0.90 & 0.56 & 19 & 0.79 & 1.36 & 0.45 & 14 \\
\hline 22 & & & & & & & & & 0.69 & 1.06 & 0.71 & 11 \\
\hline 23 & & & & & 0.43 & 0.81 & 0.43 & 13 & 0.78 & 1.45 & 0.55 & 16 \\
\hline 24 & & & & & & & & & & & & \\
\hline 25 & 0.28 & 0.42 & 0.24 & 5 & & & & & & & & \\
\hline 26 & & & & & & & & & & & & \\
\hline 27 & & & & & & & & & & & & \\
\hline 28 & & & & & & & & & & & & \\
\hline 29 & 0.18 & 0.51 & 0.24 & 5 & & & & & & & & \\
\hline 30 & 0.15 & 0.40 & 0.42 & 11 & & & & & & & & \\
\hline 31 & & & & & & & & & & & & \\
\hline 32 & & & & & & & & & & & & \\
\hline 33 & 0.20 & 0.63 & 0.37 & 6 & & & & & 0.79 & 1.28 & 0.47 & 7 \\
\hline 34 & & & & & & & & & 0.74 & 1.17 & 0.57 & 9 \\
\hline 35 & & & & & 0.46 & 0.77 & 0.83 & 12 & & & & \\
\hline 36 & & & & & & & & & & & & \\
\hline 37 & & & & & & & & & & & & \\
\hline 38 & & & & & & & & & & & & \\
\hline 39 & & & & & & & & & & & & \\
\hline 40 & & & & & & & & & & & & \\
\hline 41 & & & & & & & & & & & & \\
\hline 42 & & & & & & & & & & & & \\
\hline 43 & & & & & & & & & & & & \\
\hline 44 & & & & & & & & & & & & \\
\hline 45 & 0.17 & 0.40 & 0.41 & 81 & & & & & & & & \\
\hline 46 & 0.14 & 0.56 & 0.27 & 100 & & & & & & & & \\
\hline 47 & 0.20 & 0.50 & 0.06 & 100 & & & & & & & & \\
\hline 48 & & & & & & & & & & & & \\
\hline 49 & & & & & & & & & 0.80 & 1.48 & 0.56 & 16 \\
\hline 50 & & & & & & & & & 0.74 & 1.22 & 0.53 & 11 \\
\hline 51 & & & & & & & & & 0.81 & 1.03 & 0.51 & 9 \\
\hline 52 & & & & & & & & & 0.60 & 0.90 & 0.59 & 10 \\
\hline 53 & & & & & 0.52 & 0.94 & 0.67 & 26 & & & & \\
\hline 54 & & & & & 0.43 & 0.96 & 0.46 & 14 & & & & \\
\hline Average & 0.17 & 0.51 & 0.30 (iv) & & 0.43 & 0.82 & 0.52 & & 0.77 & 1.21 & 0.55 & \\
\hline S.D. & 0.02 & 0.08 & 0.12 & & 0.04 & 0.11 & 0.13 & & 0.06 & 0.17 & 0.07 & \\
\hline & 0.36 & 0.45 & 0.25 (Y?) & & & & & & & & & \\
\hline & 0.08 & 0.03 & 0.01 & & & & & & & & & \\
\hline
\end{tabular}

Note: $\delta=$ Isomer shift, in mm/s. Values given are $\pm 0.02 \mathrm{~mm} / \mathrm{s}$ (Dyar 1984). $\Delta=$ Quadrupole splitting, in $\mathrm{mm} / \mathrm{s}$. Values given are $\pm 0.02 \mathrm{~mm} / \mathrm{s}$ (Dyar 1984). $\Gamma=$ Peak width, in $\mathrm{mm} / \mathrm{s}$. Values given are \pm 0.02 (Dyar 1984). Area $=$ Peak area, in percent of total area. Values given are $\pm 2 \%$ of the total area (Dyar 1984). Mis (\%) = \%MISFIT (Ruby 1973). Un (\%) = Percent of uncertainty of fit (Ruby 1973). S.D. = standard deviation. Mössbauer parameters are referenced to Fe metal foil calibration.

environments that will be arbitrarily designated Y1 and Y2. These would correspond with the doublets assigned to $\mathrm{R}^{2+} \mathrm{R}^{2+}$ and $\mathrm{R}^{2+} \mathrm{R}^{3+}$ next nearest neighbors by Pieczka et al. (1997). [The phenomenum of multiple Mössbauer doublets assigned to $\mathrm{Fe}$ in a single type of site with dif- ferent populations of nearest and next nearest neighbors is also observed in other minerals such as orthopyroxene; see for example Seifert (1983).] It is noteworthy that the $\mathrm{Fe}^{2+} \mathrm{Y} 1$ sites all have very similar $\delta$ and $\Delta$ and of 1.09 $\pm 0.01 \mathrm{~mm} / \mathrm{s}$ and $2.47 \pm 0.03 \mathrm{~mm} / \mathrm{s}$, respectively. This 
TABLE 4-Continued

\begin{tabular}{|c|c|c|c|c|c|c|c|c|c|c|c|c|c|c|}
\hline \multirow[b]{2}{*}{$\delta$} & \multicolumn{3}{|c|}{$\mathrm{Fe}^{2+}$ in $\mathrm{Y} 1$} & \multicolumn{4}{|c|}{$\mathrm{Fe}^{2+}$ in $\mathrm{Y} 2$} & \multicolumn{4}{|c|}{$\mathrm{Fe}^{2+}$ in $\mathrm{Y} 3$} & \multirow{2}{*}{$\begin{array}{l}\text { Mis } \\
(\%)\end{array}$} & \multirow{2}{*}{$\begin{array}{l}\text { Un } \\
(\%)\end{array}$} & \multirow{2}{*}{$\begin{array}{l}\mathrm{Fe}^{3} \\
(\%)\end{array}$} \\
\hline & $\Delta$ & $\Gamma$ & Area & $\delta$ & $\Delta$ & $\Gamma$ & Area & $\delta$ & $\Delta$ & $\Gamma$ & Area & & & \\
\hline 1.08 & 2.46 & 0.27 & 21 & & & & & 1.04 & 1.52 & 0.67 & 25 & 0.15 & 0.020 & 54 \\
\hline 1.09 & 2.49 & 0.25 & 41 & 1.09 & 2.23 & 0.31 & 40 & 1.09 & 1.38 & 0.36 & 19 & 0.09 & 0.017 & 0 \\
\hline 1.08 & 2.46 & 0.26 & 17 & & & & & 1.04 & 1.61 & 0.65 & 21 & 0.19 & 0.021 & 62 \\
\hline \multirow[t]{2}{*}{0.87} & 1.24 & 0.65 & 35 & 1.10 & 2.46 & 0.25 & 36 & 1.08 & 2.10 & 0.39 & 29 & 0.27 & 0.022 & 18 \\
\hline & & & & & & & & & & & & 84.83 & 21.016 & 100 \\
\hline 1.09 & 2.43 & 0.25 & 20 & 1.09 & 2.13 & 0.29 & 42 & 1.03 & 1.31 & 0.58 & 33 & 0.17 & 0.037 & 6 \\
\hline 1.10 & 2.46 & 0.26 & 55 & 1.10 & 2.15 & 0.25 & 17 & 1.09 & 1.69 & 0.42 & 28 & 0.28 & 0.039 & 0 \\
\hline \multirow[t]{2}{*}{1.10} & 2.47 & 0.24 & 55 & 1.11 & 2.20 & 0.30 & 35 & 1.12 & 1.39 & 0.42 & 10 & 0.17 & 0.028 & 0 \\
\hline & & & & & & & & & & & & 6.08 & 2.655 & 100 \\
\hline 1.09 & 2.45 & 0.24 & 42 & 1.07 & 2.19 & 0.27 & 25 & 1.09 & 1.64 & 0.50 & 17 & 0.21 & 0.028 & 15 \\
\hline \multirow[t]{2}{*}{1.09} & 2.48 & 0.24 & 40 & 1.09 & 2.13 & 0.33 & 25 & 1.06 & 1.57 & 0.37 & 19 & 0.23 & 0.029 & 8 \\
\hline & & & & & & & & & & & & 0.22 & 0.027 & 100 \\
\hline 1.10 & 2.45 & 0.26 & 19 & & & & & 1.01 & 1.59 & 0.63 & 26 & 0.12 & 0.026 & 55 \\
\hline 1.09 & 2.42 & 0.28 & 22 & & & & & 1.06 & 1.55 & 0.55 & 35 & 0.21 & 0.021 & 43 \\
\hline \multirow[t]{2}{*}{1.08} & 2.43 & 0.34 & 100 & & & & & & & & & 66.65 & 14.567 & 0 \\
\hline & & & & & & & & & & & & 80.00 & 28.116 & 100 \\
\hline 1.09 & 2.49 & 0.25 & 51 & 1.09 & 2.19 & 0.31 & 24 & 1.07 & 1.66 & 0.40 & 15 & 0.08 & 0.018 & 11 \\
\hline 1.10 & 2.53 & 0.24 & 28 & 1.09 & 2.30 & 0.27 & 20 & 1.08 & 1.62 & 0.39 & 11 & 0.07 & 0.011 & 35 \\
\hline 1.09 & 2.53 & 0.24 & 36 & 1.09 & 2.33 & 0.26 & 27 & 1.05 & 1.83 & 0.47 & 19 & 0.05 & 0.019 & 15 \\
\hline 1.09 & 2.53 & 0.24 & 35 & 1.09 & 2.33 & 0.27 & 32 & 1.10 & 1.74 & 0.44 & 14 & 0.03 & 0.008 & 17 \\
\hline 1.09 & 2.47 & 0.26 & 43 & 1.05 & 2.10 & 0.30 & 12 & 1.07 & 1.52 & 0.32 & 12 & 0.18 & 0.011 & 25 \\
\hline 1.09 & 2.49 & 0.24 & 38 & 1.09 & 2.22 & 0.28 & 28 & 1.06 & 1.63 & 0.47 & 23 & 0.12 & 0.011 & 6 \\
\hline 1.10 & 2.54 & 0.25 & 41 & 1.07 & 2.30 & 0.25 & 23 & 1.09 & 1.59 & 0.25 & 8 & 0.38 & 0.057 & 21 \\
\hline 1.09 & 2.48 & 0.26 & 64 & 1.05 & 2.11 & 0.31 & 17 & 1.03 & 1.60 & 0.45 & 20 & 0.75 & 0.098 & 0 \\
\hline 1.09 & 2.49 & 0.24 & 49 & 1.08 & 2.23 & 0.26 & 19 & 1.07 & 1.62 & 0.46 & 26 & 0.24 & 0.038 & 5 \\
\hline 1.09 & 2.47 & 0.24 & 47 & 1.08 & 2.17 & 0.30 & 26 & 1.07 & 1.58 & 0.41 & 26 & 0.44 & 0.077 & 0 \\
\hline 1.10 & 2.47 & 0.24 & 40 & 1.10 & 2.17 & 0.31 & 34 & 1.05 & 1.52 & 0.55 & 26 & 0.27 & 0.021 & 0 \\
\hline 1.09 & 2.48 & 0.24 & 36 & 1.10 & 2.17 & 0.33 & 35 & 1.05 & 1.45 & 0.56 & 29 & 0.22 & 0.021 & 0 \\
\hline 1.08 & 2.49 & 0.24 & 43 & 1.09 & 2.21 & 0.28 & 23 & 1.06 & 1.66 & 0.51 & 29 & 0.08 & 0.017 & 5 \\
\hline 1.11 & 2.56 & 0.34 & 62 & 1.08 & 2.02 & 0.49 & 27 & & & & & 0.54 & 0.069 & 11 \\
\hline 1.08 & 2.45 & 0.29 & 57 & 1.12 & 2.25 & 0.31 & 36 & 1.08 & 1.40 & 0.38 & 7 & 0.16 & 0.028 & 0 \\
\hline 1.09 & 2.47 & 0.24 & 45 & 1.10 & 2.23 & 0.30 & 42 & 1.07 & 1.41 & 0.45 & 13 & 0.10 & 0.017 & 0 \\
\hline 1.10 & 2.46 & 0.24 & 44 & 1.10 & 2.16 & 0.27 & 22 & 1.08 & 1.65 & 0.45 & 21 & 0.12 & 0.011 & 10 \\
\hline 1.09 & 2.47 & 0.24 & 51 & 1.08 & 2.16 & 0.28 & 21 & 1.07 & 1.61 & 0.39 & 19 & 0.22 & 0.021 & 5 \\
\hline 1.09 & 2.48 & 0.24 & 45 & 1.08 & 2.19 & 0.28 & 20 & 1.03 & 1.65 & 0.83 & 23 & 0.26 & 0.021 & 12 \\
\hline 1.09 & 2.47 & 0.24 & 44 & 1.10 & 2.21 & 0.32 & 40 & 1.05 & 1.38 & 0.53 & 16 & 0.09 & 0.026 & 0 \\
\hline 1.09 & 2.48 & 0.26 & 49 & 1.10 & 2.26 & 0.25 & 31 & 1.15 & 1.79 & 0.61 & 20 & 0.17 & 0.037 & 0 \\
\hline 1.10 & 2.49 & 0.24 & 39 & 1.10 & 2.21 & 0.31 & 36 & 1.06 & 1.56 & 0.55 & 25 & 0.14 & 0.011 & 0 \\
\hline 1.10 & 2.46 & 0.24 & 43 & 1.09 & 2.16 & 0.29 & 29 & 1.05 & 1.51 & 0.55 & 27 & 0.20 & 0.021 & 0 \\
\hline 1.08 & 2.44 & 0.30 & 66 & 1.13 & 2.29 & 0.29 & 30 & 1.10 & 1.62 & 0.24 & 4 & 0.16 & 0.027 & 0 \\
\hline 1.09 & 2.50 & 0.24 & 46 & 1.09 & 2.22 & 0.27 & 23 & 1.08 & 1.64 & 0.50 & 31 & 0.20 & 0.037 & 0 \\
\hline 1.09 & 2.42 & 0.30 & 78 & 1.14 & 2.06 & 0.50 & 22 & & & & & 0.54 & 0.068 & 0 \\
\hline 1.08 & 2.53 & 0.26 & 30 & 1.10 & 2.31 & 0.32 & 60 & 1.09 & 1.43 & 0.47 & 10 & 0.18 & 0.028 & 0 \\
\hline & 2.52 & 0.26 & 39 & 1.10 & 2.29 & 0.29 & 47 & 1.10 & 1.62 & 0.51 & 15 & 0.12 & 0.027 & 0 \\
\hline \multirow[t]{3}{*}{1.06} & 2.43 & 0.24 & 19 & & & & & & & & & 21.17 & 4.716 & 81 \\
\hline & & & & & & & & & & & & 83.51 & 18.147 & 100 \\
\hline & & & & & & & & & & & & 96.93 & 31.216 & 100 \\
\hline 1.08 & 2.47 & 0.27 & 52 & 1.11 & 2.28 & 0.24 & 33 & 1.18 & 1.88 & 0.48 & 16 & 0.17 & 0.037 & 0 \\
\hline 1.10 & 2.43 & 0.27 & 50 & & & & & 1.11 & 1.82 & 0.50 & 35 & 0.53 & 0.051 & 8 \\
\hline 1.10 & 2.48 & 0.24 & 39 & 1.09 & 2.21 & 0.28 & 23 & 1.07 & 1.65 & 0.49 & 27 & 0.26 & 0.038 & 6 \\
\hline 1.10 & 2.43 & 0.28 & 61 & & & & & 1.05 & 1.81 & 0.50 & 30 & 0.30 & 0.031 & 5 \\
\hline 1.09 & 2.45 & 0.24 & 38 & 1.07 & 2.09 & 0.30 & 23 & 1.07 & 1.56 & 0.38 & 28 & 0.29 & 0.040 & 5 \\
\hline 1.10 & 2.47 & 0.26 & 41 & 1.03 & 2.02 & 0.34 & 22 & 0.98 & 1.46 & 0.39 & 10 & 0.25 & 0.038 & 26 \\
\hline 1.09 & 2.46 & 0.25 & 35 & & & & & 1.07 & 1.89 & 0.49 & 51 & 0.46 & 0.067 & 14 \\
\hline 1.09 & 2.47 & 0.26 & & 1.09 & 2.19 & 0.30 & & 1.07 & 1.60 & 0.48 & & & & \\
\hline 0.01 & 0.03 & 0.02 & & 0.02 & 0.08 & 0.05 & & 0.03 & 0.14 & 0.11 & & & & \\
\hline
\end{tabular}

implies that the geometry of all those sites is extremely similar from sample to sample. Because the $\mathrm{Y}$ site is dominantly divalent, this situation would be predicted by the model of Pieczka et al. (1997).

The assignment of the $\mathrm{Fe}^{2+}$ doublet with the lower $\Delta$ (average of $1.60 \mathrm{~mm} / \mathrm{s}$ ) is more problematic. As noted earlier, most previous workers assigned this doublet to ${ }^{[\mathrm{z}]} \mathrm{Fe}^{2+}$. However, in this data set, this doublet is present mainly in samples with $\mathrm{Al}>6$ atoms pfu (and $\mathrm{Si}+\mathrm{B}$ $+\mathrm{Al}>15$ ); i.e., such an interpretation would imply the unlikely scenario of $\mathrm{Fe}^{2+}$ displacing $\mathrm{Al}$ from the $\mathrm{Z}$ site. XRD work in progress (Bloodaxe et al., in preparation) also suggests that only $\mathrm{Al}$ and $\mathrm{Mg}$ occupy the $\mathrm{Z}$ site, with all iron in Y. For this reason, the lowest $\Delta \mathrm{Fe}^{2+}$ doublet must be assigned to yet another Y site (again, arbitrarily designated Y3) with different next nearest neighbors. It 

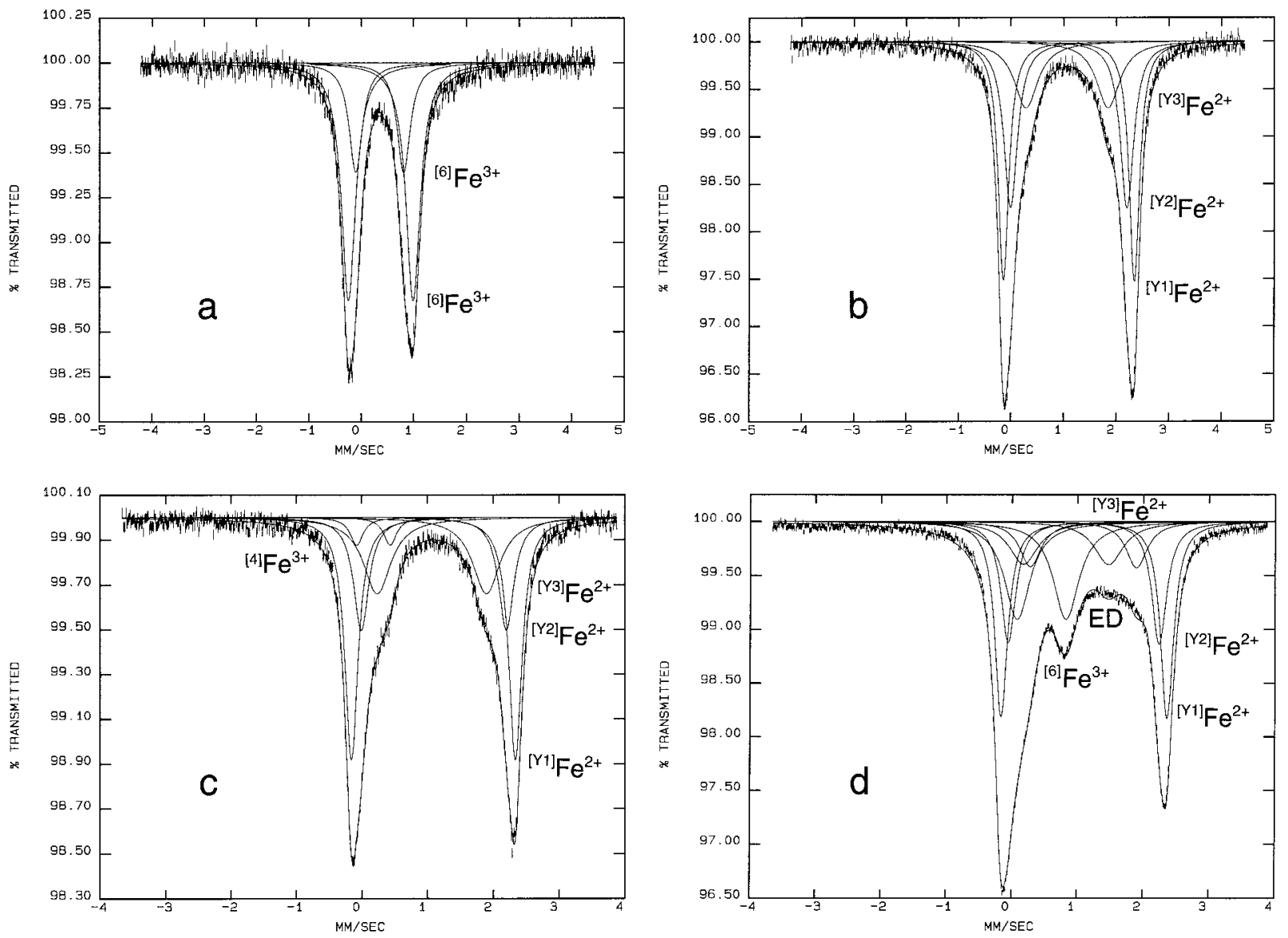

Figure 3. Mössbauer spectra of tourmaline samples from this study. (a) Buergerite sample no. 12 contains only $\mathrm{Fe}^{3+}$ as noted by Hermon et al. (1973). (b) At the other extreme, schorl sample no. 38 contains only $\mathrm{Fe}^{2+}$. (c) In schorl sample no. 29 from the Newry pegmatite, $5 \%$ of the total $\mathrm{Fe}$ is ${ }^{[4]} \mathrm{Fe}^{3+}$, while $\mathrm{Fe}^{2+}$ is distributed among the three different types of $\mathrm{Y}$ sites $(43 \%$ in $\mathrm{Y} 1,23 \%$ in $\mathrm{Y} 2$, and $29 \%$ in the Y3 site. (d) Schorl no. 19 displays peaks at 0.1 and $1.8 \mathrm{~mm} / \mathrm{s}$ that represent electron delocalization between Fe atoms in adjacent octahedra.

represents the summed contributions of the other four doublets representing ${ }^{[\mathrm{Y}]} \mathrm{Fe}^{2+}$ as assigned by Pieczka et al. (1997), which are the sites with $\mathrm{Ti}^{4+}$ next nearest neighbors or F nearest neighbors.

$\mathbf{F e}^{3+}$ doublets. The interpretation of the $\mathrm{Fe}^{3+}$ doublets is also problematic. Burns (1972) interpreted the lower $\Delta$ $(0.45 \pm 0.03 \mathrm{~mm} / \mathrm{s}$ in this data set) to represent $\mathrm{Y}$ occupancy, and the higher $\Delta(0.82 \pm 0.11 \mathrm{~mm} / \mathrm{s})$ to be $\mathrm{Z}$ occupancy; this assignment has been followed by most subsequent workers in the field (e.g., Goncharov 1997). In this data set, the intensities of the $\mathrm{Fe}^{3+}$ doublets were generally much smaller than the $\mathrm{Fe}^{2+}$ doublets, and as a result their parameters in unconstrained fits were highly variable. For this reason, it is questionable to make site assignments for $\mathrm{Fe}^{3+}$ based on the Mössbauer results, although the $\mathrm{Fe}^{3+}$ doublet areas are probably robust (see below).

This problem is well illustrated by the spectrum (Fig. $3 a)$ of the buergerite (sample no. 12). XRD refinements of this sample by Barton (1969) and Grice and Ercit (1993) suggest the presence of some $\mathrm{Fe}^{3+}$ in $\mathrm{Z}$; the latter study found $20 \%$ of the total $\mathrm{Fe}$ in $\mathrm{Z}$ and $80 \%$ in $\mathrm{Y}$. Neutron diffraction work by Tippe and Hamilton (1971) found $6.4 \%$ of the atoms in $\mathrm{Z}$ to be " $\mathrm{M}$ ", where $\mathrm{M}$ was $95 \% \mathrm{Fe}$, and magnetic susceptibility measurements of Tsang et al. (1971) estimated that $10 \%$ of the $\mathrm{Fe}^{3+}$ in that sample is in Z and $90 \%$ in Y. Thus, a Mössbauer spectrum with one large doublet corresponding to ${ }^{[\mathrm{Y}]} \mathrm{Fe}^{3+}$ and a small doublet due to ${ }^{[\mathrm{Z}]} \mathrm{Fe}^{3+}$ might be expected.

Unfortunately, this is not the case. The Mössbauer spectrum of that sample can be fit to two closely overlapping doublets with similar $\delta$ of 0.37 and $0.35 \mathrm{~mm} / \mathrm{s}$, and $\Delta$ values of 1.24 and $0.90 \mathrm{~mm} / \mathrm{s}$; peak areas are $70 \%$ and $30 \%$, respectively. If the Burns (1972) assignments are followed then both of these doublets should unequivocally be attributed to $\mathrm{Fe}^{3+}$ in the $\mathrm{Z}$ site. Another possibility is to assign the doublet with the lower $\Delta$ value to

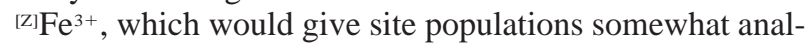
ogous to the previous work. It must also be noted that these parameters are similar to those observed by Hermon et al. (1973), who observed two doublets with $\delta=0.25$ and $0.30 \mathrm{~mm} / \mathrm{s}$ and $\Delta=1.14$ and $1.20 \mathrm{~mm} / \mathrm{s}$ (although 
they assigned them to ${ }^{\left[{ }^{\mathrm{Y}}\right]} \mathrm{Fe}^{3+}$ and ${ }^{[\mathrm{Z}]} \mathrm{Fe}^{3+}$, respectively). Clearly the Mössbauer data do not allow resolution of doublets corresponding to both ${ }^{[\mathrm{Y}]} \mathrm{Fe}^{3+}$ and ${ }^{[\mathrm{Z}]} \mathrm{Fe}^{3+}$.

For other samples in this study, the assignment of $\mathrm{Fe}^{3+}$ doublets with $\Delta \approx 0.80 \mathrm{~mm} / \mathrm{s}$ is equally difficult; compare, for example, samples no. 1 and no. 3. Mössbauer spectra of these two samples are almost identical, with prominent $\mathrm{Fe}^{3+}$ doublets at $\Delta=0.74$ and $0.77 \mathrm{~mm} / \mathrm{s}$, respectively, yet in sample no. $1 \mathrm{Si}+\mathrm{B}+\mathrm{Al} \ll 15$ (i.e., requiring $\mathrm{Z}$ site $\mathrm{Fe}^{3+}$ ), and in sample no. $3 \mathrm{Si}+\mathrm{B}+\mathrm{Al}$ $\gg 15$ (no Z site $\mathrm{Fe}^{3+}$ needed). Again, Mössbauer data do not allow the distinction between $\mathrm{Y}$ and $\mathrm{Z}$ site occupancies to be made for $\mathrm{Fe}^{3+}$. Note that XRD measurements, on a subset of 11 of these samples, do not find any $\mathrm{Fe}$ in the $\mathrm{Z}$ site; however, amounts less than about 0.20 cations per $31 \mathrm{O}$ formula unit probably would not show up. Therefore, it is not possible to use XRD refinements to understand the $\mathrm{Fe}^{3+}$ occupancies.

$\mathrm{Fe}^{3+}$ in tetrahedral coordination has the lowest values of $\delta$ and $\Delta$, with $\delta=0.17 \pm 0.02 \mathrm{~mm} / \mathrm{s}$ and $\Delta=0.51 \pm$ $0.08 \mathrm{~mm} / \mathrm{s}$. These parameters are especially convincing because they come from completely unconstrained fits, and in two cases they comprise $100 \%$ of the spectrum. Doublets representing delocalized electrons with averaged valences fall in between those assigned to $\mathrm{Fe}^{2+}$ and $\mathrm{Fe}^{3+}$ sites. For these doublets, average $\delta=0.77 \pm 0.06$ $\mathrm{mm} / \mathrm{s}$ and $\Delta=1.21 \pm 0.17 \mathrm{~mm} / \mathrm{s}$.

Four typical spectra are shown in Figure 3 for comparison. The spectrum of buergerite (sample no. 12) contains only $\mathrm{Fe}^{3+}$. Based on the above discussion, all of the $\mathrm{Fe}^{3+}$ in Figure $3 \mathrm{a}$ is assigned to ${ }^{[\mathrm{Y}]} \mathrm{Fe}^{3+}$. At the other extreme, sample no. 38 (Fig. 3b) contains only $\mathrm{Fe}^{2+}$, with occupancy in the Y1, Y2, and Y3 sites. In sample no. 29 (Fig. 3c) from the Newry pegmatite, a small amount of the total $\mathrm{Fe}$ is ${ }^{[4]} \mathrm{Fe}^{3+}$, while $\mathrm{Fe}^{2+}$ is distributed among $\mathrm{Y} 1$, Y2, and Y3. Finally, sample no. 19 (Fig. 3d) displays peaks at 0.17 and $1.47 \mathrm{~mm} / \mathrm{s}$ that constitute a doublet representing electron delocalization between $\mathrm{Fe}$ atoms in adjacent octahedra. Ferrow (1994) assigned the ED doublets in tourmaline to have $\delta$ values of $0.86,0.84$, and $0.71 \mathrm{~mm} / \mathrm{s}$ for $\mathrm{Y}-\mathrm{Y}, \mathrm{Y}-\mathrm{Z}$, and Z-Z shared electrons; therefore, we interpret the ED doublet in this sample (with $\delta$ $=0.82 \mathrm{~mm} / \mathrm{s}$ ) to represent delocalization of charge between adjacent $\mathrm{Y}$ and $\mathrm{Z}$ sites.

\section{Tourmaline compositions}

Complete compositions of all tourmaline samples in the suite are given in Table 3. Oxide data were recalculated using 31 anions and result in formulas that are charge balanced. However, in some samples this method results in stoichiometries in which the sum of cations in the sites that are known to be full from XRD data (i.e., the $\mathrm{Si}, \mathrm{B}$, $\mathrm{Y}$, and $\mathrm{Z}$ sites) is greater than the stoichiometric 18 cations. This problem cannot be blamed on "bad analyses" (although problems resulting from matrix corrections for all the light elements involved cannot be ruled out) because in many cases, our results are the averages of results from four or five different laboratories. A standardized multiple
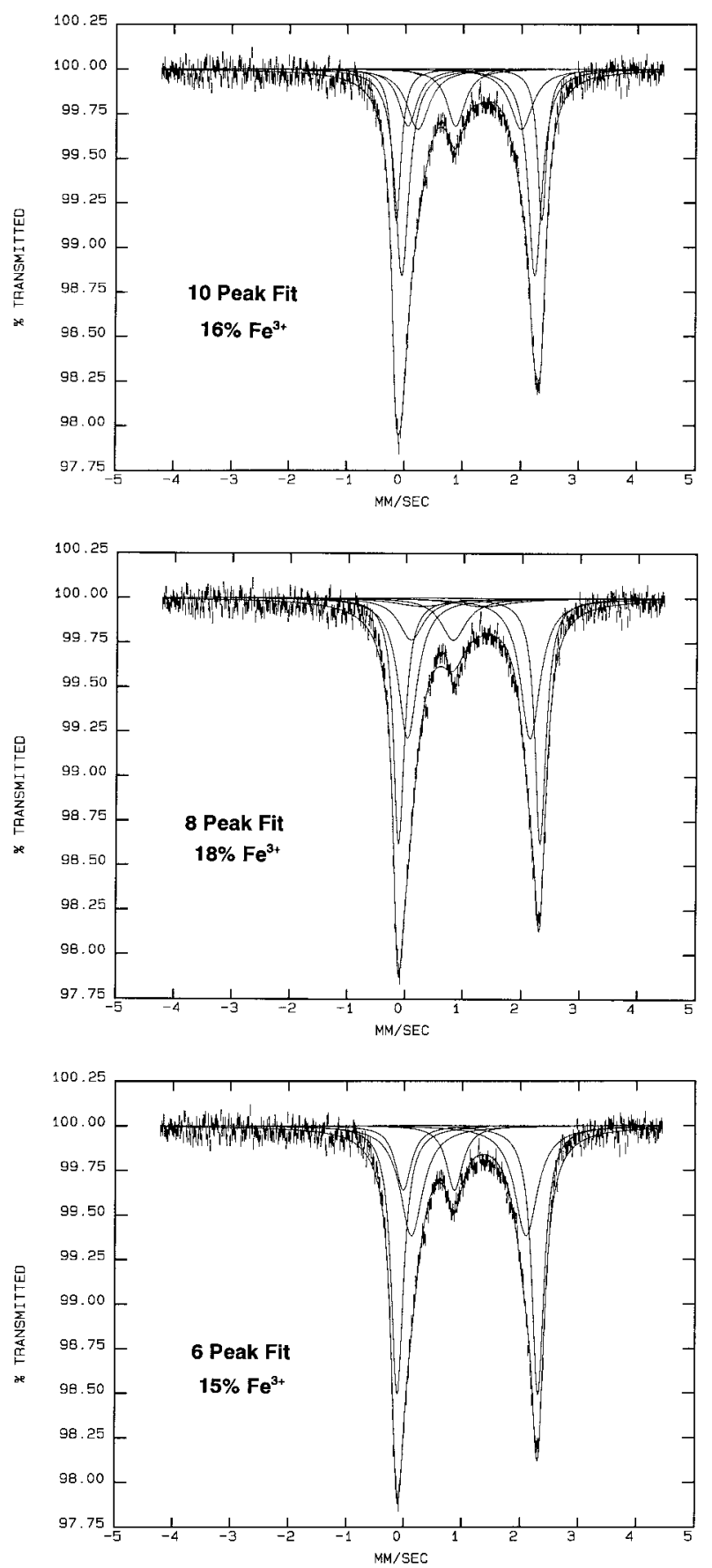

Figure 4. The $\mathrm{Fe}^{3+}$ content of tourmaline may be easily determined from Mössbauer spectra because it is independent of the fitting model used. There are 10, 8, and 6-peak fits to a single spectrum of the Jack Creek tourmaline (sample no. 10) shown here, and all the fits yield $\% \mathrm{Fe}^{3+}$ values of $16.5 \pm 1.5 \%$. Note that site occupancies, however, are very model dependent, and interpretation of spectra with varied Fe site occupancies is extremely difficult. 


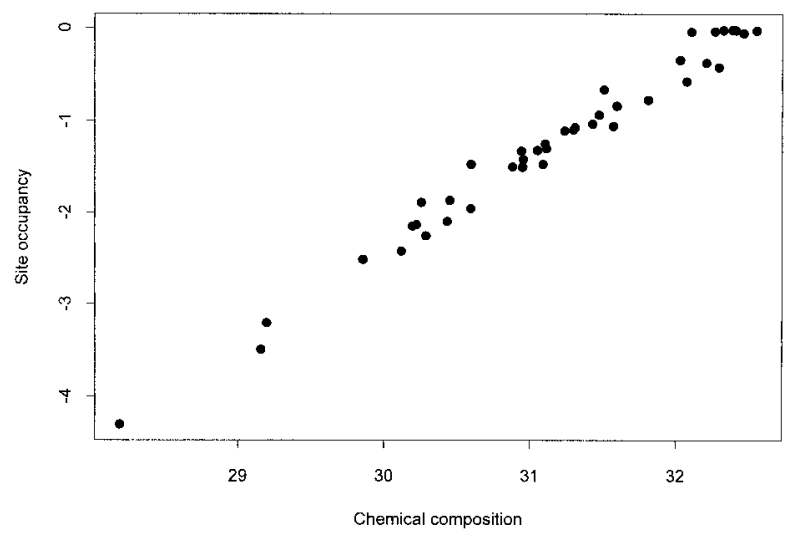

Figure 5. Plot of the first canonical variable for site occupancy vs. the first canonical variable for chemical composition $(r=0.995)$. Each canonical variate is a linear combination of the data $(\mathrm{Si}, \mathrm{Al}, \mathrm{Ti}, \mathrm{Mg}, \mathrm{Mn}, \mathrm{Ca}, \mathrm{Na}, \mathrm{K}, \mathrm{B}, \mathrm{Li}, \mathrm{F}, \mathrm{H}$ in the case of composition and ${ }^{[4 \text { or } 6]} \mathrm{Fe}^{3+},{ }^{[6]} \mathrm{Fe}^{3+},{ }^{\left[{ }_{1} 1\right]} \mathrm{Fe}^{2+},{ }^{[\mathrm{Y} 2} \mathrm{Fe}^{2+}$, and ${ }^{\left[{ }^{3} 3\right.} \mathrm{Fe}^{2+}$ in the case of site occupancy).

regression of the oxide data against the excess charge in the $\mathrm{Si}, \mathrm{B}, \mathrm{Y}$, and $\mathrm{Z}$ sites shows that "nonstoichiometry" is most affected by a combination of the variables $\mathrm{FeO}$, $\mathrm{Fe}_{2} \mathrm{O}_{3}, \mathrm{MgO}$, and $\mathrm{Li}_{2} \mathrm{O}$; i.e., no single obvious analytical variable is causing the problem. Therefore, it must be concluded that the nonstoichiometry is the result of random analytical errors propogated from the oxide measurements. The recalculated data (Table 3; and discussed below) are given with the caveat that they should not be used for comparisons where site filling is required; for such comparisons, a more valid approach is to normalize the oxide data so that the sum of cations in the $\mathrm{Si}+\mathrm{B}+\mathrm{Z}+\mathrm{Y}$ sites is equal to exactly 18 . Both methods are equally valid, and each has its advantages. In the discussion below, cations pfu for the 18 cation recalculation method will be given in parentheses following the $31 \mathrm{O}$ values.

$\mathrm{Fe}^{3+}$ contents of tourmaline range from 0 to $100 \%$ of the total $\mathrm{Fe}$, or 0.0 to 2.62 (2.62) atoms of $\mathrm{Fe}^{3+}$ per $31 \mathrm{O}$ formula unit and 0.0 to $2.41(2.34) \mathrm{Fe}^{2+}$ per $31 \mathrm{O}$ formula unit. Other than $\mathrm{Fe}$, we were most surprised to see considerable variation in the $\mathrm{Si}, \mathrm{B}, \mathrm{Li}$, and $\mathrm{H}$ contents of the tourmaline specimens studied. Si ranges from a low of 5.60 (5.57) atoms pfu to a high of 6.19 (6.05), while B varies from 2.86 (2.86) to $3.26(3.23)$ atoms pfu It is possible that $\mathrm{Si}$ atoms in excess of $3 \mathrm{~B}$ pfu must be occupying the Si site, as suggested earlier by Barton (1969) and Novozhilov et al. (1969); in fact, high B tourmaline has been synthesized, and ${ }^{11} \mathrm{~B}$ MAS NMR confirms that the excess B is in the T site (Schreyer 1997). However, the nonstoichiometry of B and $\mathrm{Si}$ is small in our samples, and is certainly within analytical error when the summed analytical errors are propogated into the formula recalculation. Therefore, our data cannot confirm B occupancy
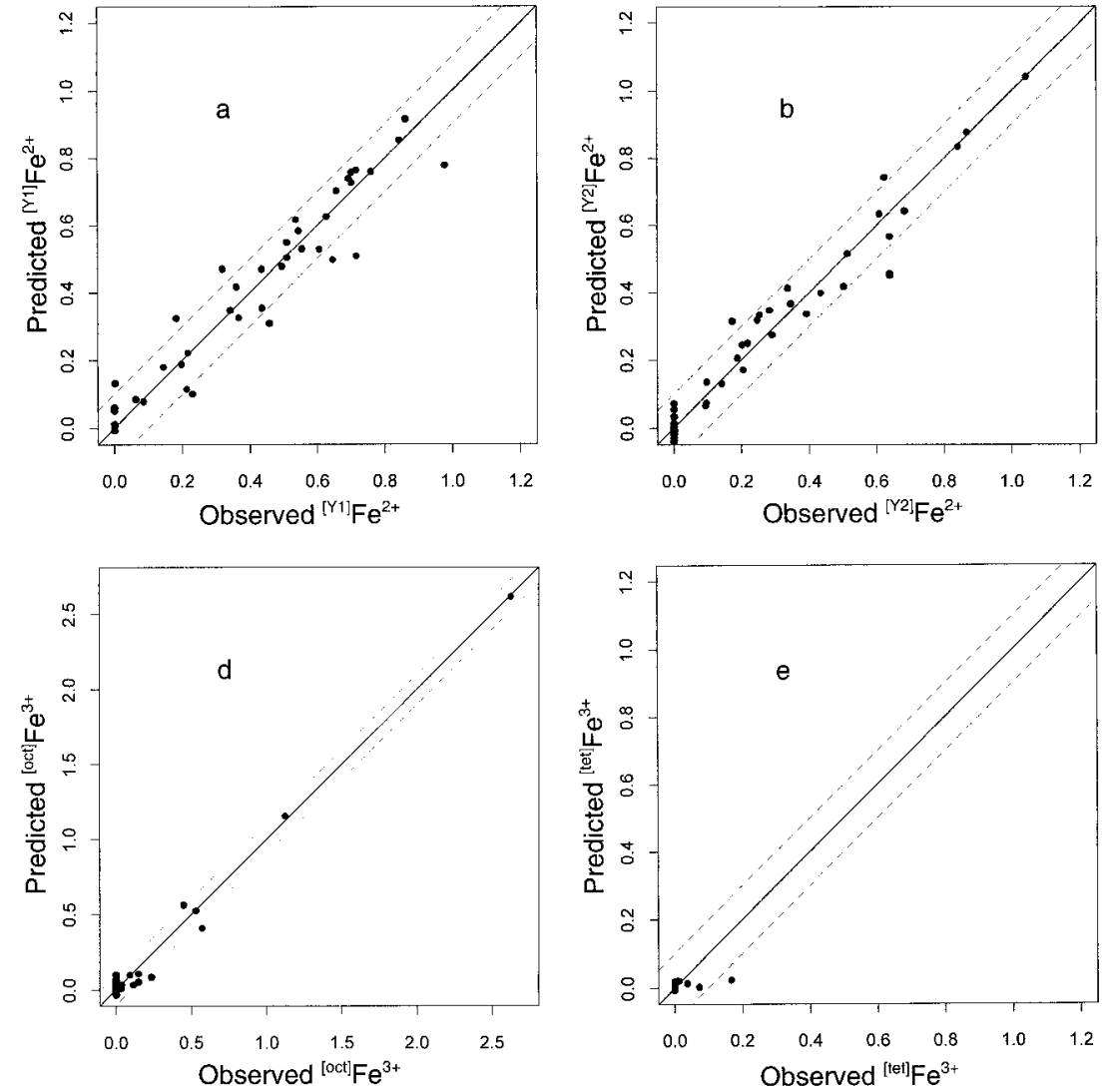

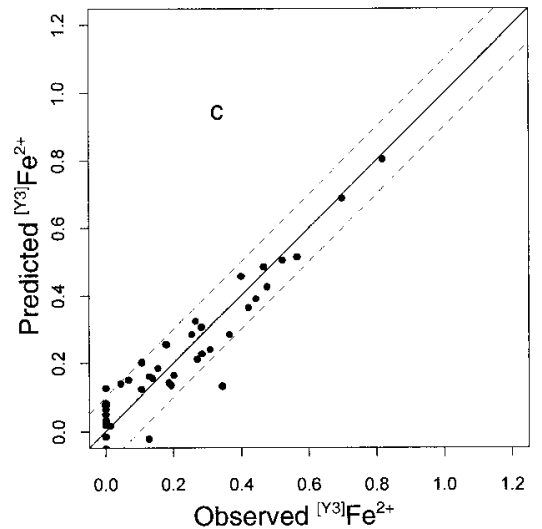

Figure 6. Comparison of observed site occupancies and the predictions of the PPR model. The solid line is the $1: 1$ line and the dashed lines indicate deviations of \pm 0.1 formula units. (a) ${ }^{\left[{ }^{1}\right]} \mathrm{Fe}^{2+}$; (b) ${ }^{\left[{ }^{2} 2\right.} \mathrm{Fe}^{2+}$; (c) ${ }^{\left[{ }^{[3}\right]} \mathrm{Fe}^{2+}$; (d) $\mathrm{Fe}^{3+}$ in octahedral sites; and (e) $\mathrm{Fe}^{3+}$ in tetrahedral or Y sites. 
TABLE 5A. Projection pursuit regression parameters

\begin{tabular}{|c|c|c|c|c|c|c|c|c|c|c|c|c|}
\hline \multirow{2}{*}{$\begin{array}{l}\text { Model } \\
\text { term }\end{array}$} & \multicolumn{12}{|c|}{ Matrix of direction vectors (a) } \\
\hline & $\mathrm{Si}$ & $\mathrm{Al}$ & $\mathrm{Ti}$ & $\mathrm{Mg}$ & $\mathrm{Mn}$ & $\mathrm{Ca}$ & $\mathrm{Na}$ & $\mathrm{K}$ & B & $\mathrm{Li}$ & $\mathrm{F}$ & $\mathrm{H}$ \\
\hline$\alpha_{1}$ & 0.5783 & -0.0827 & 0.0162 & -0.2699 & 0.0303 & 0.5601 & 0.3671 & -0.1152 & 0.0689 & -0.3029 & -0.1113 & 0.1189 \\
\hline$\alpha_{2}$ & -0.4097 & -0.1308 & -0.1301 & 0.0381 & 0.1758 & -0.2192 & 0.2633 & -0.6195 & -0.4890 & -0.0446 & 0.1119 & -0.1051 \\
\hline$\alpha_{3}$ & 0.0320 & -0.0046 & -0.0507 & -0.0316 & -0.0567 & 0.1677 & 0.1059 & 0.9663 & 0.0587 & -0.1016 & 0.0627 & 0.0374 \\
\hline$\alpha_{4}$ & 0.0413 & -0.0711 & -0.9055 & -0.0040 & 0.2927 & -0.2366 & -0.0993 & 0.1045 & 0.0365 & -0.0343 & -0.0367 & -0.0841 \\
\hline
\end{tabular}

of the Si site, although XRD does not rule out $\mathrm{B}$ occupancy of the Si site (Bloodaxe et al., in preparation). In these single crystal refinements, $\mathrm{Si}$ site occupancies of tourmaline, when released run consistently slightly low, suggesting B occupancy of that site.

Some of the tourmaline samples collected from the Black Mountain pegmatite were found to contain large amounts of $\mathrm{Li}$, up to 1.44 (1.43) atoms pfu $\mathrm{H}$ ranged from a low of $0.28(0.28)$ atoms pfu in the sample $\mathrm{Fe}^{3+}$-rich buergerite to a high of 3.76 (3.74) atoms pfu, but the majority of the samples studied were only somewhat $\mathrm{H}$ deficient (averaging 3.11 or $3.09 \mathrm{H} \mathrm{pfu}$ ). The wide variety of compositions represented in this data set makes ex- traction of the prevalent substitution vectors by inspection extremely difficult. For this reason, the intricacies of cation exchanges in this tourmaline data set will be treated separately (Lutz et al., in preparation), as noted earlier. However, some of the data involving the effects of crystal chemistry on $\mathrm{Fe}$ site partitioning will be treated below.

\section{Discussion}

\section{Dependence of $\% \mathrm{Fe}^{3+}$ on Mössbauer fitting model}

The spectra of tourmaline are difficult to fit because there can be up to six superimposed doublets represented with considerable overlap between the 12 peaks (Pieczka et al. 1997 used even more peaks in their model). For

TABLE 5B. Empirical univariate function for each term

\begin{tabular}{|c|c|c|c|c|c|c|c|}
\hline \multicolumn{2}{|c|}{ Function 1} & \multicolumn{2}{|c|}{ Function 2} & \multicolumn{2}{|c|}{ Function 3} & \multicolumn{2}{|c|}{ Function 4} \\
\hline$\alpha_{1} \cdot x$ & $\phi_{1}$ & $\alpha_{2} \cdot X$ & $\phi_{2}$ & $\alpha_{3} \cdot x$ & $\phi_{3}$ & $\alpha_{4} \cdot x$ & $\phi_{4}$ \\
\hline 2.9913 & -1.7065 & -5.2202 & -0.3165 & 0.4269 & 0.0457 & -0.6184 & -0.3175 \\
\hline 3.0142 & -1.6421 & -5.2164 & -0.3164 & 0.4287 & 0.0440 & -0.5963 & -0.6977 \\
\hline 3.0982 & -1.4069 & -5.1956 & -0.3157 & 0.4332 & 0.0401 & -0.5956 & -0.7097 \\
\hline 3.1108 & -1.3829 & -5.1203 & -0.3145 & 0.4418 & 0.0722 & -0.5879 & -0.8044 \\
\hline 3.1233 & -1.3401 & -5.0389 & -0.3180 & 0.4524 & 0.1510 & -0.5845 & -0.8174 \\
\hline 3.1583 & -1.2340 & -5.0204 & -0.3188 & 0.4688 & 0.3825 & -0.5798 & -0.7846 \\
\hline 3.1637 & -1.2104 & -5.0077 & -0.3227 & 0.4697 & 0.2827 & -0.5731 & -0.6561 \\
\hline 3.1650 & -1.2111 & -4.9891 & -0.3353 & 0.4700 & 0.2848 & -0.5611 & -0.1900 \\
\hline 3.1788 & -1.0593 & -4.9768 & -0.3428 & 0.4712 & 0.1276 & -0.5593 & -0.1066 \\
\hline 3.1925 & -0.9111 & -4.9696 & -0.3401 & 0.4730 & -0.1180 & -0.5562 & 0.1166 \\
\hline 3.2293 & -0.4467 & -4.9586 & -0.3440 & 0.4811 & -1.0612 & -0.5561 & 0.0912 \\
\hline 3.2340 & -0.4770 & -4.9488 & -0.3387 & 0.4835 & -1.0589 & -0.5552 & 0.1750 \\
\hline 3.2408 & -0.3902 & -4.9478 & -0.3408 & 0.4837 & -1.2022 & -0.5538 & 0.1991 \\
\hline 3.2581 & -0.4330 & -4.9478 & -0.3370 & 0.4895 & -0.9516 & -0.5433 & 0.4844 \\
\hline 3.2716 & -0.4383 & -4.9433 & -0.3401 & 0.4920 & -0.8578 & -0.5422 & 0.4592 \\
\hline 3.2844 & -0.4310 & -4.9423 & -0.3386 & 0.4923 & -0.8418 & -0.5371 & 0.5589 \\
\hline 3.2936 & -0.4082 & -4.9394 & -0.3373 & 0.4944 & -0.7346 & -0.5291 & 0.5674 \\
\hline 3.3146 & -0.3006 & -4.9338 & -0.3382 & 0.4961 & -0.6977 & -0.5289 & 0.5652 \\
\hline 3.3315 & -0.1998 & -4.9333 & -0.3391 & 0.4967 & -0.6722 & -0.5275 & 0.5671 \\
\hline 3.3380 & -0.1536 & -4.9236 & -0.3470 & 0.5003 & -0.5302 & -0.5223 & 0.5885 \\
\hline 3.3552 & -0.0331 & -4.9194 & -0.3498 & 0.5018 & -0.4929 & -0.5137 & 0.5254 \\
\hline 3.3605 & 0.0151 & -4.9178 & -0.3529 & 0.5021 & -0.4951 & -0.5116 & 0.4711 \\
\hline 3.3646 & 0.0397 & -4.9149 & -0.3557 & 0.5026 & -0.4740 & -0.5115 & 0.4030 \\
\hline 3.3666 & 0.0628 & -4.9126 & -0.3460 & 0.5036 & -0.4528 & -0.5109 & 0.3198 \\
\hline 3.3826 & 0.1889 & -4.9086 & -0.3435 & 0.5082 & -0.2679 & -0.5107 & 0.2517 \\
\hline 3.3910 & 0.2594 & -4.8906 & -0.2883 & 0.5096 & -0.2520 & -0.5072 & -0.3574 \\
\hline 3.3952 & 0.2920 & -4.8862 & -0.2900 & 0.5109 & -0.1974 & -0.5071 & -0.3479 \\
\hline 3.4001 & 0.3445 & -4.8754 & -0.2690 & 0.5167 & -0.1206 & -0.5060 & -0.4780 \\
\hline 3.4035 & 0.3796 & -4.8606 & -0.2727 & 0.5180 & -0.1084 & -0.5053 & -0.5331 \\
\hline 3.4421 & 0.8221 & -4.8392 & -0.2790 & 0.5190 & -0.0944 & -0.5026 & -0.6675 \\
\hline 3.4443 & 0.8376 & -4.8368 & -0.2752 & 0.5206 & -0.0676 & -0.5017 & -0.6690 \\
\hline 3.4475 & 0.8767 & -4.8313 & -0.2702 & 0.5237 & 0.0500 & -0.5002 & -0.7040 \\
\hline 3.4504 & 0.9026 & -4.8308 & -0.2440 & 0.5239 & 0.0841 & -0.4987 & -0.7001 \\
\hline 3.4567 & 0.9481 & -4.8286 & -0.2330 & 0.5247 & 0.1338 & -0.4888 & -0.6646 \\
\hline 3.4828 & 1.1376 & -4.8088 & 0.1401 & 0.5277 & 0.4720 & -0.4864 & -0.6418 \\
\hline 3.5029 & 1.2733 & -4.7879 & 0.5288 & 0.5279 & 0.5148 & -0.4771 & -0.6928 \\
\hline 3.5274 & 1.4161 & -4.7813 & 0.6277 & 0.5281 & 0.5390 & -0.4758 & -0.6110 \\
\hline 3.5398 & 1.4845 & -4.7716 & 0.7842 & 0.5289 & 0.7167 & -0.4728 & -0.6376 \\
\hline 3.5679 & 1.6422 & -4.7554 & 1.0134 & 0.5297 & 0.8485 & -0.4613 & -0.2119 \\
\hline 3.5793 & 1.7069 & -4.6751 & 2.2124 & 0.5347 & 1.8309 & -0.4195 & 1.3844 \\
\hline 3.6640 & 2.1862 & -4.4548 & 5.5042 & 0.5514 & 5.1287 & -0.3176 & 5.2725 \\
\hline
\end{tabular}


TABLE 5C. Matrix of term weights $(\beta)$

\begin{tabular}{|c|c|c|c|c|c|}
\hline $\begin{array}{l}\text { Model } \\
\text { term }\end{array}$ & {$[4$ or 6$] \mathrm{Fe}^{3+}$} & [6] $\mathrm{Fe}^{3+}$ & {$\left[\mathrm{Y}^{1}\right] \mathrm{Fe}^{2+}$} & [Y2] $\mathrm{Fe}^{2+}$ & ${ }^{[\mathrm{Y} 3]} \mathrm{Fe}^{2+}$ \\
\hline$\beta_{1}$ & -0.00806 & -0.00189 & 0.27656 & 0.23225 & 0.14691 \\
\hline$\beta_{2}$ & -0.00017 & 0.45375 & -0.11681 & -0.15794 & 0.04184 \\
\hline$\beta_{3}$ & 0.00308 & -0.06582 & -0.05083 & 0.11164 & 0.05320 \\
\hline$\beta_{4}$ & -0.00301 & -0.01526 & 0.07075 & 0.13642 & -0.06313 \\
\hline Mean $Y$ & 0.00777 & 0.14914 & 0.40777 & 0.28260 & 0.21984 \\
\hline
\end{tabular}

this reason, many different models can satisfy the conventional criteria for "good fits" based on $\chi^{2}$ and Misfit (Ruby 1973). Results of previous workers did not provide a clear consensus on the best models to be used for tourmaline; in fact, lack of agreement on the best model for tourmaline spectra undoubtedly contributes to the scatter of data points in Figure 2a. Also, some of the existing studies (e.g., Fuchs et al. 1995) should be discounted due to the fact that peak widths $(\Gamma)$ are less than the theoretical minimum imposed by the Heisenberg uncertainty principle, which is approximately $0.1940 \mathrm{~mm} / \mathrm{s}$ for ${ }^{57} \mathrm{Fe}$ (Stevens 1981). For this study, then, it was desirable that as many samples as possible be fit with the same set of constraints independent of the number of peaks involved. We sought the least constrained, most simple and realistic fits for each spectrum, which are given in Table 4. It is worth noting that approximately 20-30 "converged," statistically significant fits were obtained for each spectrum
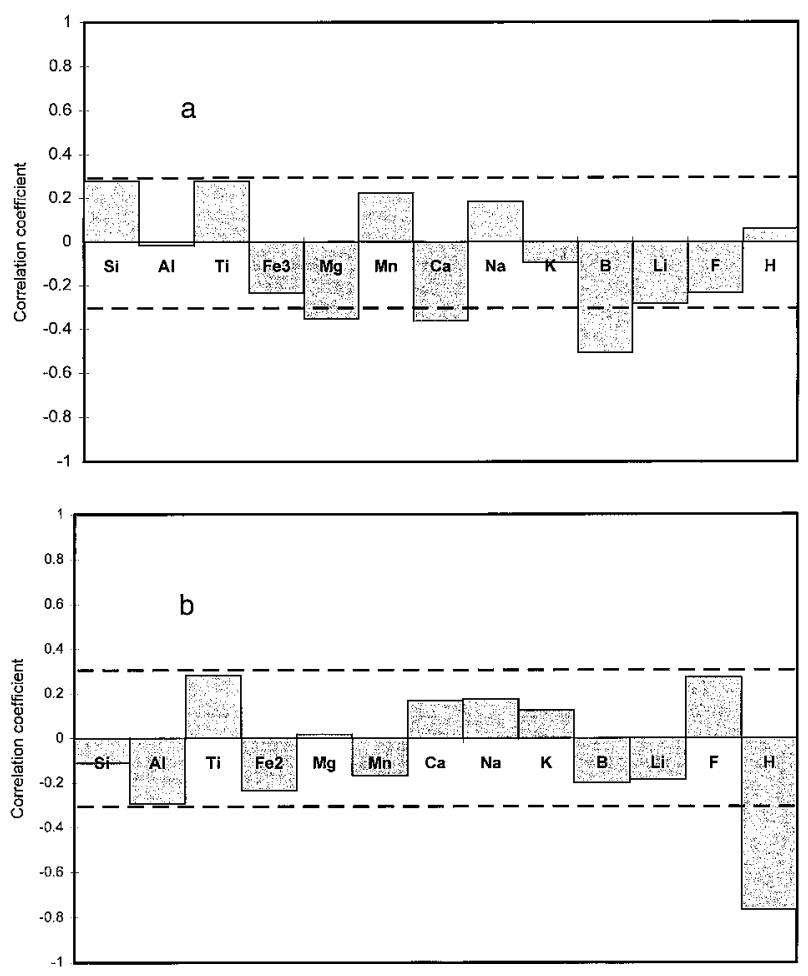

Figure 7. Linear correlation coefficients for elements with (a) $\mathrm{Fe}^{2+}$ and (b) $\mathrm{Fe}^{3+}$. Dashed lines indicate the 0.05 significance levels for the correlation coefficient based on the $t$ test. in this suite before we were able to see a systematic simple model emerge.

\section{Site distributions of $\mathrm{Fe}^{3+}$}

Because the $\mathrm{Fe}^{3+}$ peaks are generally small and heavily overlapped by the lower velocity peaks from the $\mathrm{Fe}^{2+}$ doublets, no spectrum could be fit with three doublets corresponding to $\mathrm{Fe}^{3+}$ in distinct tetrahedral, $\mathrm{Y}$, and $\mathrm{Z}$ sites. There are, however, several instances where ${ }^{[6]} \mathrm{Fe}^{3+}$ and $\mathrm{Fe}^{3+}$ in $\mathrm{ED}$ doublets are seen together, but the largest $\mathrm{Fe}^{3+}$ doublets are always octahedral. The total determined percentage of $\mathrm{Fe}^{3+}$ in tourmaline is consistently independent of the fitting model used (Fig. 4).

This report is the first study to observe doublets corresponding to ${ }^{[4]} \mathrm{Fe}^{3+}$ in tourmaline. The observed parameters fall very close to those for ${ }^{[4]} \mathrm{Fe}^{3+}$ in many other minerals as discussed above. However, we must note that it might also be possible (if somewhat unusual) for this doublet to represent ${ }^{[4]} \mathrm{Fe}^{3+}$ in the B site, as was suggested by Ja (1972) on the basis of EPR studies.

\section{Crystal chemical controls on Fe site distribution}

A relationship between chemical composition and the distribution of $\mathrm{Fe}$ among sites is revealed by canonical correlation analysis of composition ( $\mathrm{Si}, \mathrm{Al}, \mathrm{Ti}, \mathrm{Mg}, \mathrm{Mn}$, $\mathrm{Ca}, \mathrm{Na}, \mathrm{K}, \mathrm{B}, \mathrm{Li}, \mathrm{F}, \mathrm{H})$ vs. site occupancies $\left(\mathrm{Fe}^{3+}\right.$ in $\mathrm{Z}$ or $\mathrm{Y}, \mathrm{Fe}^{3+}$ in $\mathrm{Y}$ or iv, $\mathrm{Fe}^{2+}$ in $\mathrm{Y} 1, \mathrm{Fe}^{2+}$ in $\mathrm{Y} 2, \mathrm{Fe}^{2+}$ in $\mathrm{Y} 3$ ). This technique is used to investigate relationships between two different sets of variables measured on the same set of samples (Davis 1986); it finds the linear combinations of the sets of variables that give the highest correlations between the two sets. Note that Fe is not included in the composition data to reduce the possibility of forced correlations affecting the results. Samples with ED are excluded, so that 41 samples are considered in this and subsequent analyses. The results (Fig. 5) indicate a highly significant $(\mathrm{p} \ll 0.01)$ correlation $(\mathrm{r}=0.996)$. However, canonical correlation does not provide a mechanism to actually predict site occupancy from composition.

Accordingly, a model of the relationship between composition and site occupancy was developed using a nonparametric multiple regression method-projection pursuit regression (PPR) (Friedman and Stuetzle 1981) as implemented by the ppreg function in S-PLUS, version 3.3. PPR approximates the regression surface by a sum of empirical univariate functions of linear combinations of the compositions. For $N$ samples, $K$ components, $J$ sites, and $M$ model terms the regression model is 


$$
Y_{n j}=Y_{j}+\sum_{m=1}^{M} \beta_{j m} \phi_{m}\left(\alpha_{m k} \cdot X_{n k}^{m}\right)
$$

where $\mathrm{X}_{n k}$ is the $k^{\text {th }}$ component of the $n^{\text {th }}$ sample and $\mathrm{Y}_{j}$ is the mean occupancy of the $j^{\text {th }}$ site, and ynj is the predicted occupancy of the $j^{\text {th }}$ site in the $n^{\text {th }}$ sample. The parameters of the model are $\alpha_{m k}$, a matrix of direction vectors that yield linear combinations of the compositions; $\phi_{m}$, the $m^{\text {th }}$ univariate empirical function value; and $\beta_{j m}$, the weight for the $m^{\text {th }}$ model term and the $j^{\text {th }}$ site.

We obtained model predictions for site occupancy of Fe such that the ratio of the sum of squared residuals to the corrected sums of squares is 0.042. A remarkably good fit for Fe in most sites is evident (Fig. 6). The value of this PPR model is in its ability to predict Fe site occupancies and nearest neighbor populations based solely on chemical analyses. We tested the model for sensitivity to deviations of analyses from our data set by constructing and analyzing synthetic data that are randomized versions of our data. Whereas scatter about the 1:1 lines (i.e., Fig. 6) increased somewhat, as expected, no breakdown of the model was observed.

The values of $\alpha_{m k}, \phi_{m}, \beta_{j m}$, and $Y_{j}$ for our model are presented in Tables $5 \mathrm{a}-\mathrm{c}$. Note that values of the empirical functions have to be obtained by interpolation based on the values in the table. S-PLUS users can obtain the ppreg output list and a test data set by sending a diskette to Tim Lutz.

\section{Cation substitutions}

Data were studied for correlations between $\mathrm{Fe}^{3+}$ substitution and coupled substitutions on the $\mathrm{X}, \mathrm{Y}, \mathrm{Z}, \mathrm{Si}, \mathrm{B}$, and $\mathrm{OH}$ sites (Fig. 7). In no case is there an obvious relationship between $\mathrm{Fe}$ oxidation state and site charge. Thus it appears that $\mathrm{Fe}^{3+} / \mathrm{Fe}^{2+}$ ratio is controlled by the prevailing oxidation state (i.e., the supply of $\mathrm{Fe}^{3+}$ ) in the bulk rock assemblage, rather than by any particular crystal chemical substitution. There are too many coupled substitutions to be able to single out the reactions that control $\mathrm{Fe}^{3+}$. There is no single obvious crystal chemical control on $\mathrm{Fe}^{3+}$ substitution in any site.

\section{ACKNOWLEDGMenTS}

We thank Nick Foit, Joel Grice, Peter Nabalek, and Rob Kerrich for the loan of samples for this study, Jerry Delaney, Jim McGee, Anne McGuire, and Jinny Sisson for electron microprobe analyses, and LeeAnn Srogi for helpful discussions and input. The suggestions of Nick Foit and an anonymous reviewer were very helpful in revising the manuscript, as was the assistance of Charles Geiger and Anne Hofmeister. Partial support of this research was provided by NSF grants EAR-9303958 (M.D.D.), EAR9527020 (M.D.D.), and EAR-9526403 (C.V.G.) The ion microprobe data presented here were measured at the University of New Mexico/Sandia National Laboratories SIMS facility, a national multi-user facility supported in part by NSF EAR-95-06611, and we thank Michael Wiedenbeck and Grant Fowler for the analyses.

\section{REFERENCES CITED}

Alverez, M.A., Tornero, J., Vara, J.M., and Coy-Yll, R. (1975) Espectroscopia Mössbauer de variedades de turmalina. Anales de Quimica, 71, 498-502. Annersten, H. and Olesch, M. (1978) Distribution of ferrous and ferric iron in clintonite and the Mössbauer characteristics of ferric iron in tetrahedral coordination. Canadian Mineralogist, 16, 199-203.

Anovitz, L.M. and Hemingway, B.S. (1996) Thermodynamics of boron minerals: Summary of structural, volumetric, and thermochemical data. In Mineralogical Society of America Reviews in Mineralogy, 33, 181-261.

Barton, R. Jr. (1969) Refinement of the crystal structure of buergerite and the absolute orientation of tourmalines. Acta Crystallographica, B25, 1524 1533.

Belov, V.F., Kuz'min, V.I., Khimich, T.A., Dobrovol'skaya, I.V., and Shipko, M.N. (1972) Mössbauer spectroscopy of iron-rich tourmaline. Doklady Akademii Nauk SSSR, 209, 922-925.

Belov, V.F., Khimich, T.A., Shipko, M.N., Voskresenskaya, I.E., and Okulov, E.N. (1973) Gamma-resonance investigations of ferruginous tourmalines. Soviet Physics Crystallography, 18, 119-120.

Bigeleisen, J., Perlman, M.L., and Prosser, H.C. (1952) Conversion of hydrogenic materials to hydrogen for isotopic analysis. Analytical Chemistry, 24, 1356-1357.

Brown, C.D. and Wise, M.A. (1991) Mineralogy and internal structure of the Black Mountain pegmatite, Oxford County, Maine. Geological Society of America Annual Meeting, Abstracts with Programs, A329.

Burns, R.G. (1972) Mixed valencies and site occupancies of iron in silicate minerals from Mössbauer spectroscopy. Canadian Journal of Spectroscopy, $17,51-59$.

Burns, R.G. and Burns, V.M. (1984) Crystal chemistry of meteoritic hibonites. Journal of Geophysical Research, 89, C313-C321.

Dambly, M., Pollak, H., Quartier, R., and Bruyneel, W. (1976) I.R.-Irradiation enhanced effects in tourmaline. Journal of Physics, 37, C6807-C6810.

Davis, J.C. (1986) Statistics and data analysis in geology, 646 p. Wiley, New York.

Deer, W.A., Howie, R.A., and Zussman, J. (1986) Rock-forming minerals, vol. 1B: Disilicates and ring silicates, 629 p. (2nd ed.) Longman Scientific and Technical, Essex.

Delaney, J.S., Bajt, S., Sutton, S.R., and Dyar, M.D. (1996) In situ microanalysis of $\mathrm{Fe}^{3+} / \mathrm{Fe}$ in amphibole by X-ray absorption near edge structure (XANES) spectroscopy. In M.D. Dyar, C.A. McCammon, and M.W. Schaefer, Eds., Mineral spectroscopy: A tribute to Roger G. Burns, p. 165-171. Geochemical Society, Houston, Texas.

Delaney, J.S., Dyar, M.D., Sutton, S.R., and Bajt, S. (1998) Redox ratios with relevant resolution: Solving an old problem using the Synchrotron microXANES probe. Geology, 26, 139-142.

Donnay, G., Ingamells, C.O., and Mason, B. (1966) Buergerite, a new species of tourmaline. American Mineralogist, 51, 198-199.

Dyar, M.D. (1984) Precision and interlaboratory reproducibility of measurements of the Mössbauer effect in minerals. American Mineralogist, 69, 1127-1144.

Dyar, M.D. and Burns, R.G. (1986) Mössbauer spectral study of ferruginous one-layer trioctahedral micas. American Mineralogist, 71, 951-961.

Dyar, M.D., McGuire, A.V., and Ziegler, R.D. (1989) Redox equilibria and crystal chemistry of coexisting minerals from spinel lherzolite mantle xenoliths. American Mineralogist, 74, 969-981.

Dyar, M.D., Colucci, M.T., and Guidotti, C.V. (1991) Forgotten major elements: Hydrogen and oxygen variation in biotite from metapelites. Geology, 19, 1029-1032.

Dyar, M.D., Delaney, J.S., Sutton, S.R., and Bajt, S. (1996) In situ microanalysis of ferric/ferrous in geophysically important mineral groups. Abstract to Geological Society of America Annual Meeting, Denver, Colorado, A102.

Ferrow, E.A. (1993) Compositional control of plane group symmetry in tourmalines: an experimental and computer simulated TEM, crystallographic image processing, and Mössbauer spectroscopy study. European Journal of Mineralogy, 5, 479-492.

(1994) Mössbauer effect study of the crystal chemistry of tourmaline. Hyperfine Interactions, 91, 689-695.

Ferrow, E.A., Annersten, H., and Gunawardane, R.P. (1988) Mössbauer effect study on the mixed valence state of iron in tourmaline. Mineralogical Magazine, 52, 221-228.

Foit, F.F. Jr. (1989) Crystal chemistry of alkali-deficient schorl and tourmaline structural relationships. American Mineralogist, 74, 422-431.

Foit, F.F. Jr. and Rosenberg, P.E. (1979) The structure of vanadium-bearing 
tourmaline and its implications regarding tourmaline solid solutions. American Mineralogist, 64, 788-798.

Foit, F.F Jr., Fuchs, Y., and Myers, P.E. (1989) Chemistry of alkali-deficient schorls from two tourmaline-dumortierite deposits. American Mineralogist, 74, 1317-1324.

Francis, C.A., Dyar, M.D., McGuire, A.V., and Robertson, J.D. (1994) Mineral standards for geochemistry. Abstract to 16th Meeting of the International Mineralogical Association, Pisa, Italy, 124-125.

Friedman, J.H. and Stuetzle, W. (1981) Projection pursuit regression. Journal of the American Statistical Association, 76, 817-823.

Frondel, C., Biedl, A., and Ito, J. (1966) New type of ferrice iron tourmaline. American Mineralogist, 51, 1501-1505.

Fuchs, Y., Lagache, M., Linares, G., Maury, R., and Varret, F (1995) Mössbauer and optical spectrometry of selected schorl-dravite tourmalines. Hyperfine Interactions, 1995, 285-298.

Goncharov, G.N. (1997) Parameters of chemical state of iron in tourmalines as function of their forming. Abstract to Tourmaline 1997 International Symposium on Tourmaline. Nové Mesto na Morave, Czech Republic, p. $25-26$.

Gorelikova, N.V., Perfil'yev, Y.D., and Bubeshkin, A.M. (1978) Mössbauer data on distribution of Fe ions in tourmaline. International Geology Review, 20, 982-990.

Grew, E.S. (1996) Borosilicates (exclusive of tourmaline) and boron in rockforming minerals in metamorphic environments. In Mineralogical Society of America Reviews in Mineralogy, 33, 387-501.

Grice, J.D. and Ercit, T.S. (1993) Ordering of Fe and $\mathrm{Mg}$ in the tourmaline crystal structure: The correct formula. Neues Jahrbuch für Mineralogie Abhandlungen, 165, 245-266.

Henry, D.J. and Dutrow, B.L. (1990) Ca substitution in Li-poor aluminous tourmaline. Canadian Mineralogist, 28, 111-124.

(1996) Metamorphic tourmaline and its petrologic applications. In Mineralogical Society of America Reviews in Mineralogy, 33, 503-557.

Hermon, E., Simkin, D.J., Donnay, G., and Muir, W.B. (1973) The distribution of $\mathrm{Fe}^{2+}$ and $\mathrm{Fe}^{3+}$ in iron-bearing tourmalines: A Mössbauer study. Tschermaks Mineralogische und Petrographische Mitteilungen, 19, 124-132.

Hervig, R.L. (1996) Analyses of geological materials for boron by secondary ion mass spectrometry. In Mineralogical Society of America Reviews in Mineralogy, 33, 789-803.

Holdaway, M.J., Dutrow, B.L., Borthwick, J., Shore, P., Harmon, R.S., and Hinton, R.W. (1986) H content of staurolite as determined by H extraction line and ion microprobe. American Mineralogist, 71, 1135-1141.

Ja, Y.H. (1972) $\mathrm{G}=4.3$ isotropic EPR line in tourmaline. Journal of Chemical Physics, 57, 3020-3022.

King, R.W. (1990) Tourmaline from mesothermal gold deposits of the Superior Province, Canada. Textural, chemical, and isotopic relationships, 228 p. $\mathrm{Ph} . \mathrm{D}$. thesis, University of Saskatchewan, Saskatoon.

Korovushkin, V.V., Kuzmin, V.I., and Belov, V.F. (1979) Mössbauer studies of structural features in tourmaline of various genesis. Physics and Chemistry of Minerals, 4, 209-220.

Kraczka, J., Pieczka, A., Hrynkiewicz, A.Z., and Zabinski, W. (1986) Mössbauer study of tourmalines. Hyperfine Interactions, 29, 1121-1124.

Lanford, W.A. (1992) Analysis for hydrogen by nuclear reaction and energy recoil detection. Nuclear Instruments and Methods, B66, 65-82.

Linares, J., Fuchs, Y., Lagache, M., and Robert, J.L. (1996) A Mössbauer study of synthetic tourmaline. Conference Proceedings, I. Ortailli, Ed., International Conference on Applications of the Mössbauer Effect, Bologna, $50,785-788$.

London, D., Morgan, G.B. VI, and Wolf, M.B. (1996) Boron in granitic rocks and their contact aureoles. In Mineralogical Society of America Reviews in Mineralogy, 33, 299-329.

Long, G.J., Cranshaw, T.E., and Longworth, G. (1983) The ideal Mössbauer effect absorber thickness. Mössbauer Effect Reference and Data Journal, 6(2), 42-49.

Mason, B., Donnay, G., and Hardie, L.A. (1964) Ferric iron tourmaline from Mexico. Science, 144, 71-73.

Mattson, S.M. and Rossman, G.R. (1984) Ferric iron in tourmaline. Physics and Chemistry of Minerals, 11, 225-234.

McGee, J.J. and Anovitz, L.M. (1996) Electron probe microanalysis of geo- logic materials for boron. In Mineralogical Society of America Reviews in Mineralogy, 33, 771-788.

McGuire, A.V., Dyar, M.D., and Ward, K.W. (1989) The neglected $\mathrm{Fe}^{3+} / \mathrm{Fe}^{2+}$ ratio: a study of $\mathrm{Fe}^{3+}$ contents of megacrysts from alkali basalts. Geology, $17,687-689$.

McKlveen, J.W. (1981) Fast neutron activation analysis: Elemental data base, 286 p. Ann Arbor Science Publications, Ann Arbor, Michigan.

Novozhilov, A.I., Voskresenskaya, I.E., and Samoilovich, M.I. (1969) E.P.R. study of tourmalines. Kristallografiya, 14, 507-509.

Pieczka, A. and Kraczka, J. (1997) Thermal oxidation of $\mathrm{Fe}^{2+}$ ions in the schorl-dravite series and its significance in the analysis of distribution of $\mathrm{Fe}^{2+}$ octahedral ions. Abstract to Tourmaline 1997 International Symposium on Tourmaline, Nové Mesto na Morave, Czech Republic, 72-73.

Pieczka, A., Kraczka, J., and Zabinski, W. (1997) Mössbauer spectra of $\mathrm{Fe}^{3+}$ poor schorls: reinterpretation of the spectra on a basis of an ordered structure model. Abstract to Tourmaline 1997 International Symposium on Tourmaline, Nové Mesto na Morave, Czech Republic, p. 74-75.

Pollak, H. and Bruyneel, W. (1974) Saut d'électrons et le rapport $\mathrm{Fe}^{++} / \mathrm{Fe}^{+++}$ dans deux silicates. Journal of Physics, 35, C6571-C6574.

Rockhold, J.R., Nabelek, P.I., and Glascock, M.D. (1987) Origin of rhythmic layering in the Calamity Peak satellite pluton of the Harney Peak Granite, South Dakota: The role of boron. Geochimica et Cosmochimica Acta, 51, 487-496.

Rossman, G.R., Grew, E.S., and Dollase, W.A. (1982) The colors of sillimanite. American Mineralogist, 67, 749-761.

Ruby, S.L. (1973) Why Misfit when you already have $\chi^{2}$ ? In I.J. Gruverman and C.W. Seidel, Eds., Mössbauer Effect Methodology, 8, p. 263-276. Plenum Press, New York.

Saegusa, N., Price, D.C., and Smith, G. (1979) Analysis of the Mössbauer spectra of several iron-rich tourmalines (schorls). Journal de Physique, 40, C2-456-C2-459.

Schreyer, W. (1997) Experimental studies on tourmaline end-members. Abstract to Tourmaline 1997 International Symposium on Tourmaline, Nové Mesto na Morave, Czech Republic, p. 89-90.

Schwartz, K.B., Nolet, D.A., and Burns, R.G. (1980) Mössbauer spectroscopy and crystal chemistry of natural Fe-Ti garnets. American Mineralogist, 65, $142-153$.

Seifert, F. (1983) Mössbauer line broadening in aluminous orthopyroxenes: Evidence for next nearest neighbors interactions and short-range order. Neues Jahrbuch für Mineralogie Abhandlungen, 148, 141-162.

Scorzelli, R.B, Baggio-Saitovitch, E., and Danon, J. (1976) Mössbauer spectra and electron exchange in tourmaline and staurolite. Journal of Physics, 37, C6801-C6805.

Simon, H.F. (1973) Near-infrared and Mössbauer study of cation site occupancies in tourmalines, 67 p. M.S. thesis, Massachusetts Institute of Technology, Cambridge, Massachusetts.

Smyth, J.R. and Bish, D.L. (1988) Crystal Structures and Cation Sites of the Rock-Forming Minerals, 332 p. Allen and Unwin, Boston.

Smyth, J.R., Madel, R.E., McCormick, T.C., and Munoz, J.L. (1990) Crystalstructure refinement of a F-bearing spessartine garnet. American Mineralogist, 75, 314-318.

Snetsinger, K.G. (1966) Barium-vanadium muscovite and vanadium tourmaline from Mariposa county, California. American Mineralogist, 51, 16231639.

Stevens, J.G. (1981) Useful constants. In J.W. Robinson, Ed., Handbook of Spectroscopy III, p. 404-406. CRC Press, Boca Raton, Florida.

Taylor, M.E. (1996) The crystal chemistry of iron in tourmaline. M.S. thesis, 138 p. West Chester University, West Chester, Pennsylvania.

Tippe, A. and Hamilton, W.C. (1971) A neutron-diffraction study of the ferric tourmaline, buergerite. American Mineralogist, 56, 101-113.

Tsang, T., Thorpe, A.N., Donnay, G., and Senftle, FE. (1971) Magnetic susceptibility and triangular exchange coupling in the tourmaline mineral group. Journal of Physics and Chemistry of Solids, 32, 1441-1448.

Volborth, A. and Banta, H.E. (1963) Oxygen determination in rocks, minerals, and water by neutron activation. Analytical Chemistry, 35, 2203-2205.

ManusCRiPt ReCeIVed November 8, 1996

Manuscript accepted February 26, 1998

Paper handled by Charles A. Geiger 